\title{
Vibrational Diagnostics of Rotating Machinery Malfunctions
}

\author{
AGNES MUSZYNSKA \\ Senior Research Scientist \& Research Manager, Bently Rotor Dynamics Research Corporation, Minden, Nevada, U.S.A.
}

(Received January 13, 1994)

\begin{abstract}
This paper outlines rotating machinery malfunction diagnostics using vibration data in correlation with operational process data. The advantages of vibration monitoring systems as a part of preventive/predictive maintenance programs are emphasized. After presenting basic principles of machinery diagnostics, several specific malfunction symptoms supported by simple mathematical models are given. These malfunctions include unbalance, excessive radial load, rotor-to-stator rubbing, fluid-induced vibrations, loose stationary and rotating parts, coupled torsional/lateral vibration excitation, and rotor cracking. The experimental results and actual field data illustrate the rotor vibration responses for individual malfunctions. Application of synchronous and nonsynchronous perturbation testing used for identification of basic dynamic characteristics of rotors is presented. Future advancements in vibration monitoring and diagnostics of rotating machinery health are discussed. In the Appendix, basic instrumentation for machine monitoring is outlined.
\end{abstract}

Key Words: Vibration diagnostics of machine malfunctions; Vibration monitoring on machinery; Plant maintenance; Rotating machinery; Rotor/bearing/seal systems; Modal testing; Dynamic stiffness identification; Expert systems.

C ompetitiveness of the world market stimulates a plant's main goal of increased throughput of highquality products. This goal is often achieved by increased machine speeds and reduced machinery outages, which, in turn, put a great emphasis on machinery health. Vibration monitoring as a part of preventive/predictive maintenance programs assists in achieving the main goal, and has proven to be highly cost effective. The benefits from vibration monitoring include the following:

- Reduced production losses.

- Enhanced efficiency, reliability, availability, and longevity of machinery.

- Reduced maintenance costs (reduced overtime payments for labor, decreased spare parts and stocked inventory costs, reduced fuel costs).

- Improved work load planning.

- Enhanced safety and environmental programs.

The core of machine vibration monitoring schemes consists of appropriately selected and strategically lo- cated transducers for capturing machine vibration and process data. Advancements in electronics have considerably improved data management systems (Fig. 1). A new generation of electronic transducers, and computers with their large memories, fast processor speeds, high quality graphics, standard communication links, and advanced software based on intimate knowledge of solid/fluid dynamics and thermodynamics, provide the capability to extract reliable and meaningful information from an increased volume of data in given amounts of time and budget. Vibration and process data presented in convenient cross-correlated formats permit effective and reliable evaluation of the machinery health, give early warning of impending failures, and allow determination of the cause of malfunctions. Faults detected early enough can be monitored closely to ensure that significant damage is avoided, and that the machine is operated safely until the maintenance can be scheduled to correct the malfunctions.

The basic principles creating the foundation for machine malfunction diagnosis methodology applicable for 


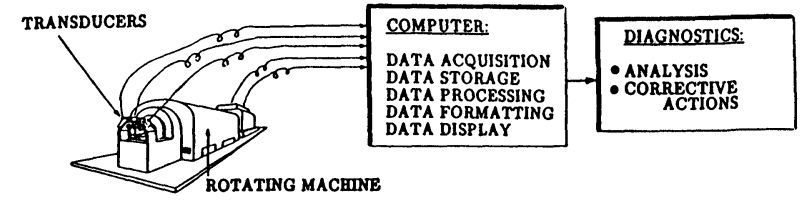

FIGURE 1 Machine diagnostic system.

both periodic and continuous monitoring are (Laws et al [1987]):

- Know the basic mechanical, fluidic, thermal, and electric (as applicable) characteristics of the machine.

- Know the types of malfunction mechanisms and their corresponding symptoms that the machine is likely to incur.

- Monitor the key parameters that will indicate a change in condition of the machine.

- Reduce diagnostic data into interpretable formats and compare the results with analytical predictions.

- Understand the historical and related events that may have caused changes in machine condition.

- Present data on the machine condition in as solid engineering form as possible.

- Take prompt action based on the machine diagnosis.

Any machine health condition evaluation requires the knowledge of the fundamental physical characteristic of the machine, such as geometry, weight, clearances, etc. However, understanding the basic factors of the machine goes beyond knowing these characteristics. It involves understanding the machine's dynamic behavior during its operation. To achieve this goal, the design data should be accessible to the machine user, and the process and vibration data need to be acquired as early as during acceptance testing and commissioning. Various methods, such as controlled unbalance response testing (synchronous perturbation) and nonsynchronous perturbation testing, are used to document the rotor response during steady-state (on-line with partial and full load) and transient (start-up and shutdown) conditions. This process provides baseline data for insight to:

- The natural frequencies of the system and mode shapes, especially those of the machine train rotor.

- The amount of effective damping in the system.

- The margin of safety against instability of a rotor system.

- The load-to-vibration relationships.

- Acceptable vibration limits.
Analytical models of the machine dynamic behaviorparticularly the models based on a modal approach-are of tremendous value in future diagnostic procedures. On large critical machines or machines that have had a history of vibration problems, it is desirable to incorporate these analytical models into the machine's database. The analytical and testing baseline information is necessary for comparing the machine's previous and present condition when a machine malfunction occurs. It can also be used after the problem is fixed to verify the validity of the correction.

Most of the baseline data and process/operational requirements, as well as a comprehensive prediction profile of the machine, should be provided by the machine manufacturer. Also, a monitoring system, or at least the recommendation of transducer locations for diagnostic purposes, should be included as a part of the design for all rotating machines.

Monitoring of the machine operation and its process variables should be cross-correlated with a simultaneous monitoring of the machine vibrational behavior. The operation of machines is a dynamic process, and the process data, to be of significance, must include dynamic components of the quantities monitored. Changes in process or auxiliary variables (e.g., changes in bearing temperature or changes in driving electric motor power consumption) may represent the first warning signals of impending vibration problems. Machine vibrations, being symptoms of malfunctions, occur in response to either input forcing function changes or machine dynamic stiffness (transfer function) changes. Vibrations also carry information about the character of, and possible source of, the malfunctions. Both process and vibration data should be trended, ideally in cross-correlated formats using whichever cross-correlations that yield pertinent information.

While fulfilling the main function of the rotating machines, the machinery rotors are most prone to mechanical vibrations, and are the principal source of vibrations. Most malfunctions originate from direct transfer of rotor rotational energy into vibrational energy of various modes. Among the latter, the lateral modes of the rotor are of the highest concern. Most often they represent the lowest modes of the machine structure. Rotor vibrations are eventually transmitted to pedestals, casing, and foundation. It is evident that measuring vibrations "at the source" becomes vital for correct evaluation of the machine health. That is why the generally accepted practice is to utilize two noncontacting displacement transducers installed in orthogonal XY configuration on or near each radial bearing to measure lateral vibrations and static centerline position of the rotor relative to the mounting fixture. Vibration measure- 
ment on pedestals or casing by using velocity pickups and/or accelerometers provides indirect information about the vibration source. The clarity of this information depends on the mechanical transmissibility of elements between the source and transducers. In addition, these transducers are (i) not able to measure shaft centerline position, (ii) do not indicate the direction of shaft orbiting, (iii) do not provide information on the rotor mode shape, and (iv) their sensitivity in the most important low frequency range is very poor. Housing vibration measurements can be recommended only for noncritical, general purpose low-speed machines.

In the USA the American Petroleum Institute has adopted a recommended practice (RP) entitled "Vibration, Axial Position, and Bearing Temperature Monitoring System" (RP\#670). It outlines the system requirements for installing proximity transducers in the XY configuration on compressors and their drivers to observe shaft motion. In addition to these radial transducers, this recommended practice calls for two axially oriented noncontacting proximity transducers. These probes are used to monitor and alert about machine thrust problems, and are often tied to automatic trip when a danger condition exists. Both of these transducer installation practices are also appropriate for the monitoring and protection of turbogenerators, pumps, fans, and other rotating machines.

One of the very important transducers called for in the $\mathrm{RP}$ is a phase angle reference transducer, the Keyphasor ${ }^{\circledR}$ transducer, which provides a rotor once-per-turn marker. It is used to monitor rotative speed and phase of rotor $1 \times$ (synchronous) response, as well as phases of the rotative frequency fractional and/or multiple type vibration components. The Keyphasor ties the rotor vibration data to its rotational motion. The information provided is extremely valuable for balancing, as well as in diagnosing various machine malfunctions.

Six vibration-related parameters are commonly measured on rotating machines under steady-state (on-line) and transient (start-up and shutdown) conditions:

- Overall magnitude of vibration for indicating presence and severity of a problem.

- Frequencies of vibration components for insight into the root causes of the malfunction.

- Timebase waveforms and orbital paths of rotor lateral motion (rotor orbital and its direction) for insight into the nature of the malfunction. The orbits are especially significant: they represent magnified snapshots of the rotor actual motion.

- Amplitudes and phase angles of the orbital motion components of the rotor. The phase angle is one of the most important parameters for rotating machine mal- function identification. Phase angles of vibration components with synchronous frequency, twice rotative frequency, and of whirling and whipping frequencies are especially meaningful.

- Shaft centerline position for insight into the radial load status, and into the specific location of the rotor with respect to the stationary components of the machine.

- The ratio of overall to the $1 \times$ vibration amplitudes, as a signal for operators to get machinery diagnostics assistance.

In the next section several rotating machine vibration malfunctions, with their symptoms and simple mathematical models explaining malfunction physical principles, will be discussed.

\section{DIAGNOSIS OF PARTICULAR MALFUNCTIONS OF ROTATING MACHINES ILLUSTRATED BY BASIC MATHEMATICAL MODELS OF THE ROTOR}

Widespread availability of computers accelerated the appearance of more and more sophisticated software for calculations of various aspects of rotating machine dynamics. Finite Element Method allows computation of the machine's solid structure response, including fluid interactions. Complex computer models built from separate blocks are, however, only as good as the basic particular model's adequacy to real, observable phenomena occurring in rotating machines. Research on the latter is still pending. During the last twenty years, the widespread application of vibration supervisory systems on machines has accumulated broad case history data on major vibrational problems occurring in specific types of machines. The implementation of the acquired theoretical and practical knowledge into the machine design procedure is, however, slow and seldom consistent. As a result, the machines often contain an intrinsic dynamic incorrectness, and the machine performances are seldom smooth since the beginning of their operation. The dynamic process of further operation inevitably leads to worsening of the performance. Maintenance engineers who have been taught machine dynamics on computers have difficulty in understanding basic physical phenomena affecting the machine behavior. Quick fixes are based on trial and error methods. And yet, if the machine vibrates, there always exists a cause of it.

The purpose of this section is to present simple mathematical models which describe fundamental cause/ effect relationships for several, most important vibrationrelated machinery malfunctions. In all models the modal 
approach is adopted, and mainly the first lateral mode of the rotor is considered. Further generalizations of this approach are obvious.

\section{Unbalance: Residual and Controlled}

Unbalance is a most common malfunction in rotating machines. Unbalance in the rotating machine is a condition of unequal mass distribution at each section of the rotor. In an unbalanced condition, the rotor mass centerline does not coincide with the axis of rotation. During rotation, rotor unbalance generates an inertia centrifugal force which rotates at the rotor rotational frequency. Unbalance represents then the first, fundamental mechanism to transfer the rotational energy into vibrations. The mathematical model of an isotropic unbalanced rotor (rotating at a constant speed $\Omega$ in a counterclockwise direction) at its first lateral mode is as follows:

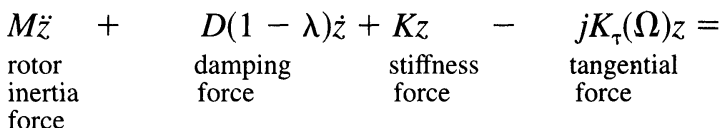

$$
\begin{aligned}
& \underset{\text { unbalanced }}{m r \Omega^{2}} e^{j(\Omega t+\delta)}, z=x+j y,,^{\bullet}=d / d t, j=\sqrt{-1} \\
& \text { excitation force }
\end{aligned}
$$

The notations are given in the Nomenclature. Eq. (1) represents the balance of forces acting on the rotor, including the tangential force oriented in the direction of rotation. In most cases the tangential force is a function of the rotative speed, and represents another mechanism transferring energy of rotation into vibrations. The factor $1-\lambda$ emphasizes the rotor damping reduction effect due to fluid tangential forces (see Section 2.5). The left side of Eq. (1) describes the rotor restraints, its dynamic stiffness. At the right side, the inertia unbalance force creates the forcing function. The effect of it, i.e., the rotor forced response as observed by two XY transducers, is characterized by the circular orbital (precessional) motion in the direction of rotation (following the force), with frequency $\Omega$, amplitude $A$, and phase lag $\alpha$. This orbital motion observed by only one lateral displacement transducer will represent a sinusoidal waveform. The forced solution of Eq. (1), i.e., the rotor response, is:

$$
z=A e^{j(\Omega t+\alpha)}
$$

When substituted to Eq. (1), it yields

$$
\begin{aligned}
& {\left[K-M \Omega^{2}+j D(1-\lambda) \Omega-j K_{t}\right] A e^{j \alpha}=m r \Omega^{2} e^{j \delta}} \\
& \text { rotor synchronous } \\
& \text { dynamic stiffness } \\
& \begin{array}{ll}
\text { synchronous } & \text { unbalance } \\
\text { response } & \text { vector } \\
\text { vector } &
\end{array}
\end{aligned}
$$

Eq. (3) illustrates the balance of causes and effects: The rotor response depends on both its dynamic stiffness and unbalance excitation. Any change in the rotor response may, therefore, result from either a change in unbalance or a change in the restraining rotor dynamic stiffness components (e.g., shaft crack would reduce $K$ ).

The elements of the synchronous response vector (amplitude and phase angle) can easily be calculated from Eq. (3):

$$
\begin{aligned}
& A=\frac{m r \Omega^{2}}{\sqrt{\left(K-M \Omega^{2}\right)^{2}+\left[D(1-\lambda) \Omega-K_{\tau}\right]^{2}}} \\
& \alpha=\delta-\arctan \frac{D(1-\lambda) \Omega-K_{\tau}}{K-M \Omega^{2}}
\end{aligned}
$$

It is clear that when the rotative speed $\Omega$ reaches the value $\sqrt{K / M}$, the response amplitude will exhibit a resonance peak, as it will only be controlled by the relatively small damping term, additionally weakened by the tangential component $K_{\tau}$. At resonance the response phase $\alpha$ differs from its original value $\delta$ by $-90^{\circ}$. The rotative speed $\Omega=\sqrt{K / M}$ is classically referred to as a critical speed, or the first balance resonance speed; $\sqrt{K / M}$ represents a system natural frequency, the lowest of the rotor lateral modes.

The synchronous response vector locus, from the data provided by one lateral displacement transducer during rotor start-up or shutdown, are usually presented in the Bode and/or polar plot formats. For this purpose, the rotor vibration signal is filtered to the frequency of rotation, and usually compensated by subtracting the slow roll (low frequency) $1 \times$ response vector. These formats are widely used for unbalance diagnosis and corrections (Fig. 2). They also allow for easy detection of resonant frequencies, and evaluation of the system effective damping and synchronous amplification factor using the half-power bandwidth method.

The unbalance malfunction is corrected by routine procedures of machine balancing.

\section{Synchronous and Nonsynchronous Perturbation Testing for System Identification}

Eq. (3) represents the basic equation not only for unbalance diagnosis, but also for identification of the synchronous dynamic stiffness of the rotor by using synchronous perturbation technique (Muszynska et al. [1989a]). This method consists of inputting a controlled unbalance to the rotor and measuring its synchronous response. From three components of Eq. (3), now the dynamic stiffness can be calculated: 


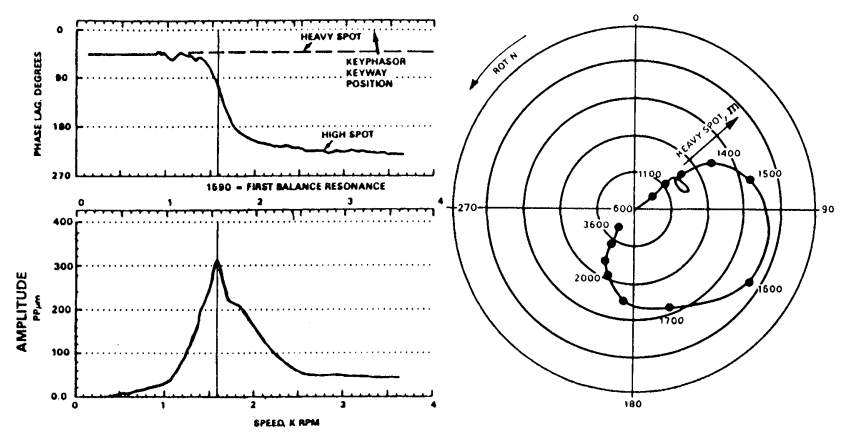

FIGURE 2 Bode and polar plots of a machine rotor $1 \times$ filtered response measured by a vertical displacement transducer. These formats help to identify the first balance resonance frequency, and the unbalance (heavy spot) location.

$$
\begin{aligned}
& \text { Direct Synchronous Dynamic Stiffness }= \\
& K-M \Omega^{2}=\left[m r \Omega^{2} \cos (\delta-\alpha)\right] / A
\end{aligned}
$$

Quadrature Synchronous Dynamic Stiffness =

$$
D(1-\lambda) \Omega-K_{\tau}=\left\{m r \Omega^{2} \sin (\delta-\alpha)\right] / A
$$

By plotting the results versus rotative speed, the observed $^{1}$ modal stiffness, mass, and damping can be identified $^{2}$ (Fig. 3). The procedure of synchronous perturbation is routinely used in the calibration weight balancing methodology. The "influence vectors" (elements of the transfer function) represent elements of the inverse of the dynamic stiffness (in the matrix sense). During balancing, their meaningfulness is most often underestimated. When stored and compared after consecutive balancing of the machine, they will reflect changes in the rotor restraint characteristics through the machine life span. They would assist in early detection of such malfunctions as cracked shaft, rubs, and loose parts.

Easy to perform, the synchronous perturbation provides very useful, but usually insufficient, data for appropriate identification of the machine system parameters, especially those which vary with the rotative speed. More sophisticated, nonsynchronous perturbation testing yields the required data (Muszynska, [1986a],

\footnotetext{
"The term "observed" refers to the fact that the obtained parameters usually differ from actual modal ones, due to specific axial locations of measuring transducers, and controlled unbalances.

${ }^{2}$ In order to eliminate the effect of the rotor residual unbalance, the controlled unbalance for the first run in inserted at the angular local $\delta$, for the second run at the location $\delta+180^{\circ}$, then the synchronous response vectors from these runs are vectorially subtracted, producing net response to controlled unbalance (Muszynska et al. [1989a].
}

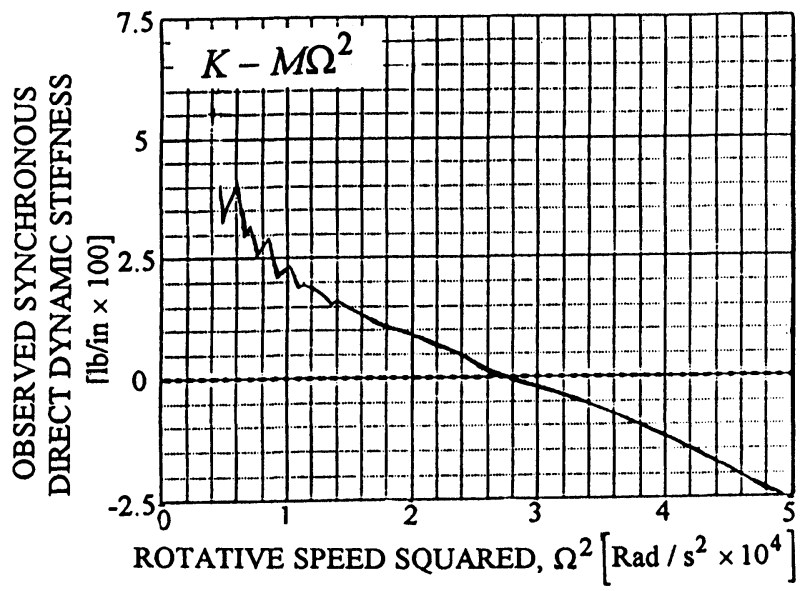

FIGURE 3 Observed synchronous direct dynamic stiffness of a rotor: identification of modal mass $M$ and stiffness. $K$ (Muszynska et al. [1989a]).

Muszynska et al. [1990a]). In one of possible schemes, the rotor rotating at a constant speed is excited by a nonsynchronously rotating external force, generated by an auxiliary shaker-like device. The sweep frequency response data for a sequence of constant rotative speeds provide the necessary information for the identification of the rotor basic rotative speed dependent characteristics.

The nonsynchronously excited rotor model and its response are as follows:

$$
\begin{aligned}
& M \ddot{z}+D(1-\lambda) \dot{z}+K z-j K_{\tau}(\Omega) z=F e^{j\left(\omega t+\delta_{1}\right)}, \\
& z=A e^{j(\omega t+\alpha)}
\end{aligned}
$$

where the amplitude and phase of the response can be calculated as:

$$
\begin{gathered}
A=\frac{F}{\sqrt{\left(K-M \omega^{2}\right)^{2}+\left[D(1-\lambda) \omega-K_{\tau}(\Omega)\right]^{2}}}, \\
\alpha=\delta_{1}-\arctan \frac{D(1-\lambda) \omega-K_{\tau}(\Omega)}{K-M \omega^{2}}
\end{gathered}
$$

It can easily be shown that independently from the resonance, as previously occurring at $\omega=\sqrt{K / M}$ and referred to as "direct" mechanical resonance, there exists a "quadrature" resonance when $\omega=K_{\tau}(\Omega) / D(1-\lambda)$. The onset of rotor instability occurs when both resonant frequencies coincide: $\omega=\sqrt{K / M}=K_{\tau}(\Omega) /$ $D(1-\lambda)$. The denominator of Eq. $\left(5_{1}\right)$ becomes zero. For nonresonant frequencies, the actual frequency difference $K_{\tau}(\Omega) / D(1-\lambda)-\sqrt{K / M}$ represents a meaningful measure of the machine stability margin (Muszynska et al. [1990a]). 
For the sweep-frequency perturbation, the dynamic stiffness components are calculated in the entire frequency range:

Direct Dynamic Stiffness $=$

$$
K-M \omega^{2}=F \cos \left(\delta_{1}-\alpha\right) / A
$$

Quadrature Dynamic Stiffness $=$

$$
D(1-\lambda) \omega-K_{\tau}(\Omega)=F \sin \left(\delta_{1}-\alpha\right) / A
$$

Using this technique for a sequence of constant rotative speeds, all rotative speed dependent components can be identified. This, in particular, applies to the tangential force. ${ }^{3}$

The concept of perturbation technique is explained here on a simple rotor model. It can, however, be extended to more complex cases used for identification of several mode modal parameters (Muszynska et al. [1989a]). The nonsynchronous perturbation testing should become a routine procedure during initial testing of the machine performance.

\section{Misalignment and Radial Load on the Rotor}

Following unbalance, the misalignment of machine train rotors is the second most common malfunction of rotating machinery. The literature on rotor unbalance malfunction and corrective balancing procedures can be found in thousands of papers, books, and reports. Amazingly, misalignment has not drawn that much researchers' attention. The literature on rotor misalignment malfunction is very scarce. Machine alignment procedures now involving quite sophisticated laser-optic instrumentation are considered routine. Handbooks on "how to align a machine train" are popular. There is, however, very little published on the misalignment malfunction, its destructive, overloading effects on rotors and bearings, or on how to diagnose misalignment by using vibration monitoring.

One of the main effects of misalignment between rotors in the machine train is a generation of rotor load in a specific radial direction. The misalignment causes a constant radial force which pushes the rotor to the side. The same result can be caused by a strong radial component of the fluid flow in fluid-handling machines, especially evident in single volute pumps, or in turbines during partial steam admission on the first-stage nozzles.

\footnotetext{
${ }^{3}$ The gyroscopic effect, another rotative speed dependent component, was not included in the considered here rotor model. The nonsynchronous perturbation applied to an overhung rotor with a strong, gyroscopic effect provides identification of pertaining parameters (Muszynska, et al. [1986a].
}

Gravitational load on horizontal rotors, thermal expansion load, offset, or cocked bearing-related load, and gear mesh forces also belong to this category. Due to the radial force, the rotor becomes displaced from the original position, and moved to higher eccentricity ranges inside the bearing and seals. It may also become bowed, and rotate in a bow configuration. At these conditions the nonlinear effects of the system become active. A simple rotor model extended from Eq. (1) describes this phenomenon:

$$
\begin{aligned}
& M \ddot{x}+D_{x}\left(1-\lambda_{x}\right) \dot{x}+K_{x} x+K_{\tau x}(\Omega) y=m r \Omega^{2} \cos (\Omega t \\
& +\delta)+P \cos \gamma
\end{aligned}
$$

$\mathrm{M} \ddot{y}+\mathrm{D}_{y}\left(1-\lambda_{y}\right) \dot{y}+K_{y} y-K_{\tau y}(\Omega) x+y\left(K_{n} y+\ldots\right)=$ $m r \Omega_{2} \sin (\Omega t+\delta)+P \sin \gamma$

where $P$ represents the load force applied to the rotor in the radial direction at the reference angle $\gamma$ and $K_{n}$ is a generalized nonlinear stiffness coefficient. Eq. (6) contains, therefore, the stiffness-related nonlinear term, which represents only an example of nonlinearities which may be active in the system (e.g., fluid film radial stiffness and damping at high eccentricities have different functional form of nonlinearities). This term may be referred to as the first term of the Taylor expansion for any nonlinear function. The dots indicate possible existence of other nonlinear terms.

Due to nonlinearity, the unbalance-forced response of the rotor will now contain not only the synchronous component (2), but also its higher harmonics: $2 \times, 3 \times$, etc., vibration components. The radial force also causes the shaft average position to be affected by nonlinearity.

Excessive radial load can be diagnosed from the vibrational data. Abnormal orbital motion, abnormal radial average shaft centerline position, and abnormal shaft versus casing motion are indicative of excessive radial loads. Figure 4 illustrates how an orbital motion can be analyzed. As the orbit changes from an elliptical shape to a "banana" shape, it is an indication that higher frequency components are present. The analysis of the shaft centerline position should also be used to diagnose excessive loads. A combination of shaft centerline position and orbits reflects the actual situation at each bearing. In Figure 4, the radial load on the shaft forces the shaft down in one bearing and up in the other. Note the elliptical and "banana" shape nature of the orbits as a result of an increasing load. The Keyphasor marks on the orbits (Fig. 4b) indicate that, although the bearings are loaded in opposite directions, both ends of the shaft vibrate in phase. Since at higher eccentricity positions nonlinear effects are present, the increasingly rich spectrum of higher harmonics will be an indication of the excessive radial loads. Axial measurements may addi- 

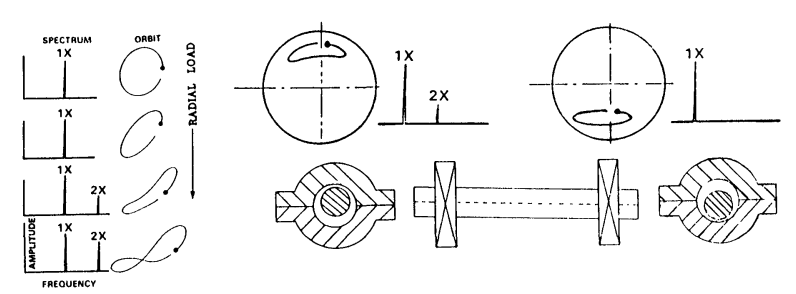

FIGURE 4 Excessive radial load/misalignment diagnosis: frequency spectra, orbits, and centerline positions.

tionally indicate an impending misalignment problem on the machine.

It should be well understood that a continuously acting radial load on a rotor operating at a constant speed causes periodic $1 \times$ frequency (plus possible higher harmonic components) reversal stresses in the rotor fibers. For a high speed continuously operating machine, the number of the stress reversal cycles may swiftly reach the fatigue limit (especially when the load results in high stress amplitudes, leading to low cycle fatigue). The vibrational and rotor centerline position data have to be analyzed from the point of view of the rotor stress; very often a lower lateral vibration amplitude signifies higher rotor load, and resulting harsher stress conditions on the rotor. This may lead to rotor cracking and its premature failure.

\section{Rotor-to-Stator Rubbing}

Rubbing between the rotor and stationary part of the machine is a serious malfunction that may lead to a catastrophic failure. Rubbing involves several physical phenomena, such as friction, stiffening/coupling effect, impacting, and may affect solid/fluid/thermal balance in the machine system. Rubbing always occurs as a secondary effect of a primary malfunction, such as unbalance, misalignment, or fluid-induced, self-excited vibrations, which all result in high lateral vibration amplitudes and/or changes in the shaft centerline position within available clearances.

The first two rub-related phenomena mentioned above are relatively easy to model. The original one-lateralmode model (Eq. (1)) is completed now with the second equation representing the balance of forces at the rubbing location (Fig. 5):

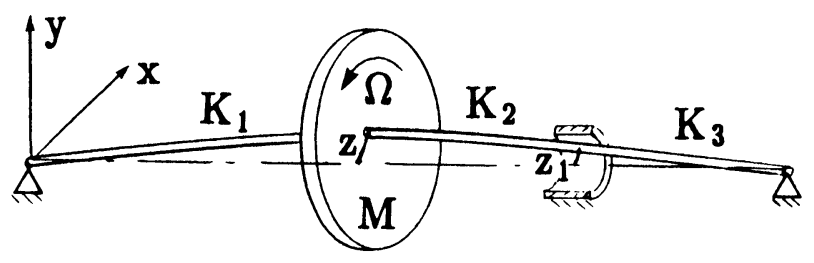

FIGURE 5 Physical model of the rotor.

$$
\begin{aligned}
& M \ddot{z}+D\left(1-\lambda_{R}\right) \dot{z}+\left(K_{1}+K_{2}\right) z-K_{2} z_{1} \\
& =m r \Omega^{2} e^{j(\Omega t+\delta)}+P e^{j \gamma}, \\
& \left(K_{2}+K_{3}\right) z_{1}-K_{2} z+v K_{2}\left(\left|z_{1}\right|-c\right)(1+j \mu) \frac{z_{1}}{\left|z_{1}\right|}=0, \\
& v=\left\{\begin{array}{l}
0 \text { for }\left|z_{1}\right|<c \\
1 \text { for }\left|z_{1}\right| \geq c
\end{array}\right. \\
& \quad\left|z_{1}\right|=\sqrt{x_{1}^{2}+y_{1}^{2}}
\end{aligned}
$$

The model (7) reflects an intermittent action of rub; the system becomes piece-wise continuous with variable stiffness. The modal stiffness $K$ is now split into three sections (see Fig. 5); $K_{s}$ represents the generalized stiffness of the stator at the rubbing location. The latter is involved in the system dynamics during a fraction of rotor vibration period (occasionally $v=1$ ). Friction is approximated by using the Coulomb's model. The friction force is oriented in the tangential direction opposite to the direction of rotation. The impacting effect is not included in this model, as it requires considerations of the local contact area dynamics. It must, however, be included in extended models used for numerical calculations. The effect of short-lasting, impact-related rotor/ stator contact can be considered in terms of rotor free vibration response, following each impact (Muszynska [1984a]). For very light partial rubs on one location, at rotative speeds exceeding double value of the first balance resonance speed, these free vibrations superposed on $1 \times$ unbalance forced vibrations result in fractional subsynchronous vibrations: $1 / 2 \times, 1 / 3 \times$, etc., most often with backward directions, resulting in external loops on the rotor orbits (Figs. 6 and 7). The adjustment of frequency from the free response to fractional frequency occurs during the rotor/stator contact period, and it is due to the unbalance force/response phase relationship at each specific speed. In another way of reasoning, due to system nonlinearity, the unbalance force excites fractional frequency responses.

The model (7) can also be used to demonstrate that the rubbing may cause self-excited vibrations (known as dry whip or full annular rub, occurring mainly in seals) independently from the excited vibrations. When $m r=0$, $P=0$ and $v=1$ continuously, the solution of Eq. (7)

$$
z=B e^{j(\omega t+\beta)}, \quad z_{1}=B_{1} e^{j \omega t}
$$

describes these self-excited circular vibrations with amplitudes $B, B_{1}$, frequency $\omega$ and relative phase $\beta$ (Muszynska [1984b]). The latter quantities can be calculated from four algebraic equations obtained after sub- 


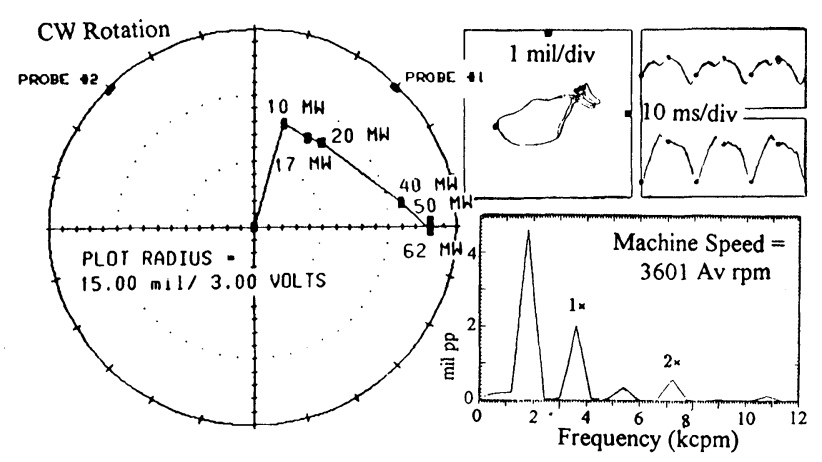

FIGURE 6 Rub diagnosis in a turbogenerator: shaft centerline as function of load; orbit, timebase waveform, and spectrum at $50 \mathrm{MW}$ and $3600 \mathrm{rpm}$. The presence of rub-induced $1 / 2 \times$ subsynchronous vibrations (BN MDS Reports).

stituting Eqs. (8) into Eqs. (7). The rotor disk vibration relative phase depends on the rotor characteristics. The disk amplitude is a function of the latter, and the rotor amplitude at the seal:

$$
\begin{aligned}
& \beta=\arctan \frac{-\omega D\left(1-\lambda_{R}\right)}{K_{1}+K_{2}-M \omega^{2}}, \\
& B=\frac{K_{2} B_{1}}{\sqrt{\left(K_{1}+K_{2}-M \omega^{2}\right)^{2}+\left[D\left(1-\lambda_{R}\right) \omega\right]^{2}}}
\end{aligned}
$$

The frequency equation yields four self-excited frequencies $\omega$ with approximate values: $\pm \sqrt{\left(K_{1}+K_{2}\right) / M}$ (a "short" rotor natural frequency) and

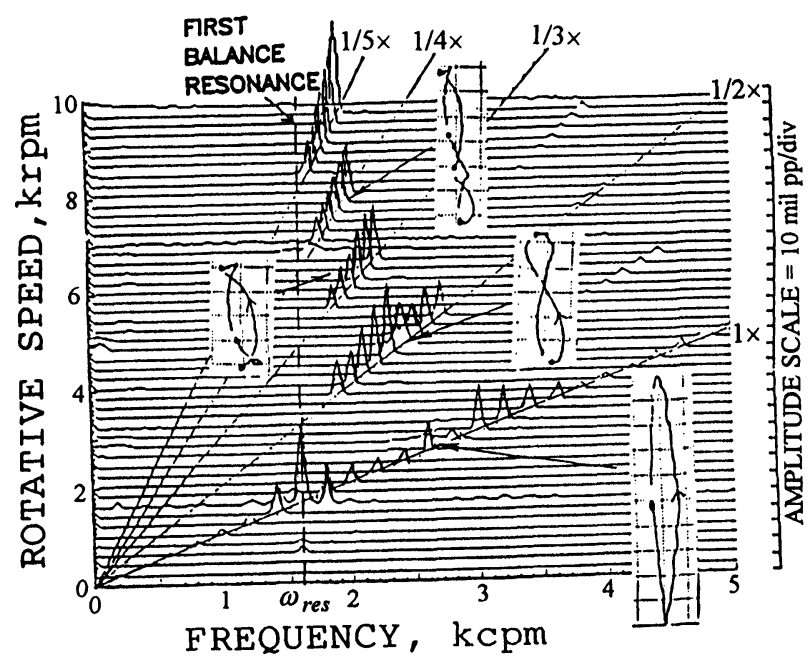

FIGURE 7 Spectrum cascade of rotor vibrations and corresponding orbits. Rotor is lightly rubbing on the upper surface of a seal (Muszynska [1984a]). $\pm \sqrt{\left[K_{1}+K_{2} K_{3} /\left(K_{2}+K_{3}\right)\right] / M}$ (full rotor natural frequency). Finally the fourth algebraic equation provides the amplitude $B$ for each frequency $\omega$. If the stator (or seal) is infinitely stiff, then $B_{1}=c$. During dry whip, the rotor rolls while sliding against the seal in the direction opposite to the direction of rotation while the other sections of the rotor vibrate at resonant conditions of the backward mode, independently of rotative speed (Fig. 8). Most often observed frequency of the dry whip is - $\sqrt{\left(K_{1}+K_{2}\right) / M}$. High radial (normal) and corresponding friction (tangential) forces at the contacting surfaces may lead to extremely severe damage of the seal and rotor surfaces in a very short time. In addition, due to the backward mode of vibrations, the rotor operates under severe alternating stress with relatively high frequency $\Omega+\omega$. The rub-related failures of machines occur quite often (Muszynska [1989b]).

Since model (7) includes the rub-generated nonlinear term, the rotor responses, besides the fundamental components, contain a spectrum of higher harmonics. The impacting, as another nonlinear mechanism of rubbing (not included in the model (7)), increases the strength of this spectrum even more. Rotor-to-stationary element rubbing is actually a very rich phenomenon resulting in rapidly changing system parameters with a tendency to chaotic motions, thus the model (7) represents only the simplest first approach, which includes the basics. (The effects of rub on the torsional load, thermal, and fluid flow-related effects were neither included here.)

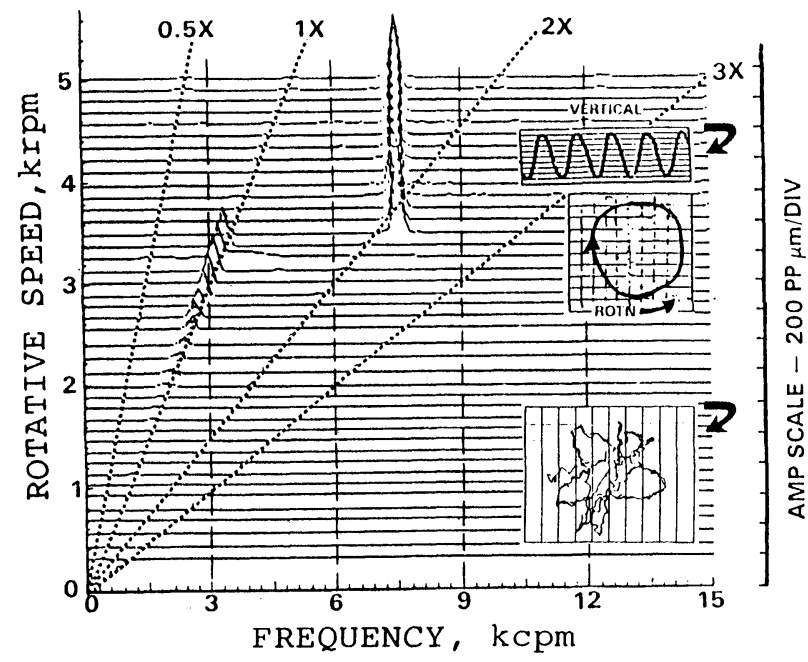

FIGURE 8 Spectrum cascade of vertical vibrations of a rotor rubbing inside the seal, timebase waveform, and orbits at selected speeds. In the lower speed range, rotor bounces inside the seal, producing multiple higher harmonics of $1 \times$. At higher speed a full annular rub (dry whip) occurs (Muszynska [1984b]). 
The diagnosis of rotor rubbing from vibrational data is based on (i) changes in rotor synchronous response vectors, (ii) appearances of subsynchronous fractional components which, when filtered, most often exhibit backward orbiting, (iii) short-lasting appearances of components with natural frequencies (due to transient character of rubbing), (iv) appearances of higher harmonics of the fundamental components, and (v) changes in shaft centerline position data.

Partial or fully backward orbiting of the rotor is the most characteristic for rubs, distinguishing this malfunction from the others.

The coupling/stiffening effect of rubbing can be detected through changes in the balance resonant frequencies. It is especially noticeable in differences between the rotor start-up and shutdown synchronous responses, when rubbing occurs in the range of the balance resonance speeds (Fig. 9). The amplitudes become limited at rubbing location; they may increase, however, in other shaft sections; phase lags less. Thermal effect of rubbing, causing at certain rotative speeds an ever-changing thermal bow-related unbalance of the shaft, may lead to $1 \times$ amplitude fluctuations and continuous $1 \times$ phase lagging as a function of time resulting in characteristic spirals (Fig. 10).

Diagnosis of the rub axial location requires an analysis of vibration component phases along the rotor in correlation to the particular modes. For the first mode it can be based on Eqs. (9).

\section{Fluid-Induced Instabilities}

Rotating shafts operate in a fluid environment. An interaction between the rotor and the surrounding fluid becomes significant if the clearances between rotating

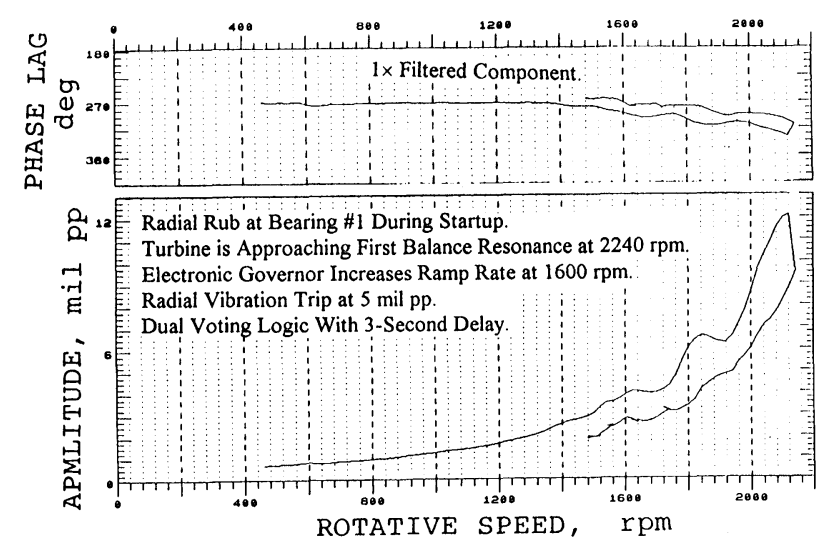

FIGURE $91 \times$ Bode plot of a rubbing turbogenerator rotor during start-up and shutdown after tripping (BN MDS Reports). and stationary parts are small, and the rotors operate at low eccentricity within these clearances. Due to friction, shaft rotation generates a circumferential flow of the fluid. The fluid, in turn, produces a dynamic effect: rotating forces acting in feedback at the rotor. Such situations take place in lightly loaded fluid-lubricated bearings, in seals, in balance pistons, in stator/blade tip clearances, and rotor/stator peripheries. Similar phenomena occur in all cases where the fluid becomes involved in rotational motion, such as in centrifuges, rotors with trapped fluid, or rotors filled with fluid. Well known are the final dynamic effects of such rotor/fluid interaction, namely, the rotor self-excited subsynchronous vibrations of the "fluid whirl" and "fluid whip" type.

The simplest mathematical model of the rotor (concentricity rotating inside a bearing or seal) which describes the fluid whirl/whip phenomena is as follows (Fig. 5) (Muszynska [1986b]):

$$
\begin{gathered}
M \ddot{z}+D\left(1-\lambda_{R}\right) \dot{z}+\left(K_{1}+K_{2}\right) z-K_{2} z_{1}=0 \\
\left(K_{2}+K_{3}\right) z_{1}-K_{2} z+D_{b}(\dot{z}_{1}-\underbrace{j \lambda \Omega z_{1}}_{\text {tangential force }})+ \\
\left(K_{b}+K_{n b}\left|z_{1}\right|^{2}+\ldots\right) z_{1}=0
\end{gathered}
$$

Eq. $\left(10_{2}\right)$ includes fluid film radial damping $D_{b}$ and fluid nonlinear stiffness $K_{b}+K_{n b}\left|z_{1}\right|^{2}+\ldots$ at the bearing (or seal) location. It also includes fluid average circumferential velocity ratio $\lambda$, the measure of the circumferential flow strength; $\lambda \Omega$ is the rate at which the fluid damping force rotates (Fig. 11) (Muszynska [1986b, 1988a, 1990a]). For $K_{3} \neq 0$, the model represents a rotor/seal or stator/rotor/blade tip clearance system (Fig. 5); when $K_{3}$ $=0$, it represents a rotor/bearing system. In Eqs. (10) the unbalance, radial load, and other forces are omitted for clarity. Note that, in contrast with the rubbing case, the fluid-induced tangential force is oriented in the direction of rotation.

The solution (8) of Eqs. (10) describes the rotor self-excited vibrations of the fluid whirl or whip type. The amplitudes $B, B_{1}$, frequencies $\omega$, and the relative phase $\beta$ can be calculated by substitution (8) into Eqs. (10). $B$ and $\beta$ remain the same as in Eqs. (9). The frequency $\omega$ and amplitude $B$ are calculated from the real and imaginary parts of the following equation (only the first nonlinear term included):

$$
\begin{aligned}
& {\left[K_{2}+K_{3}+K_{b}+D_{b} j(\omega-\lambda \Omega)+K_{n b} B^{2}\right] \times} \\
& {\left[K_{1}+K_{2}-M \omega^{2}+j D\left(1-\lambda_{R}\right) \omega\right]-K_{2}^{2}=0}
\end{aligned}
$$

It can be shown that there exist two frequencies which satisfy Eq. (11): 


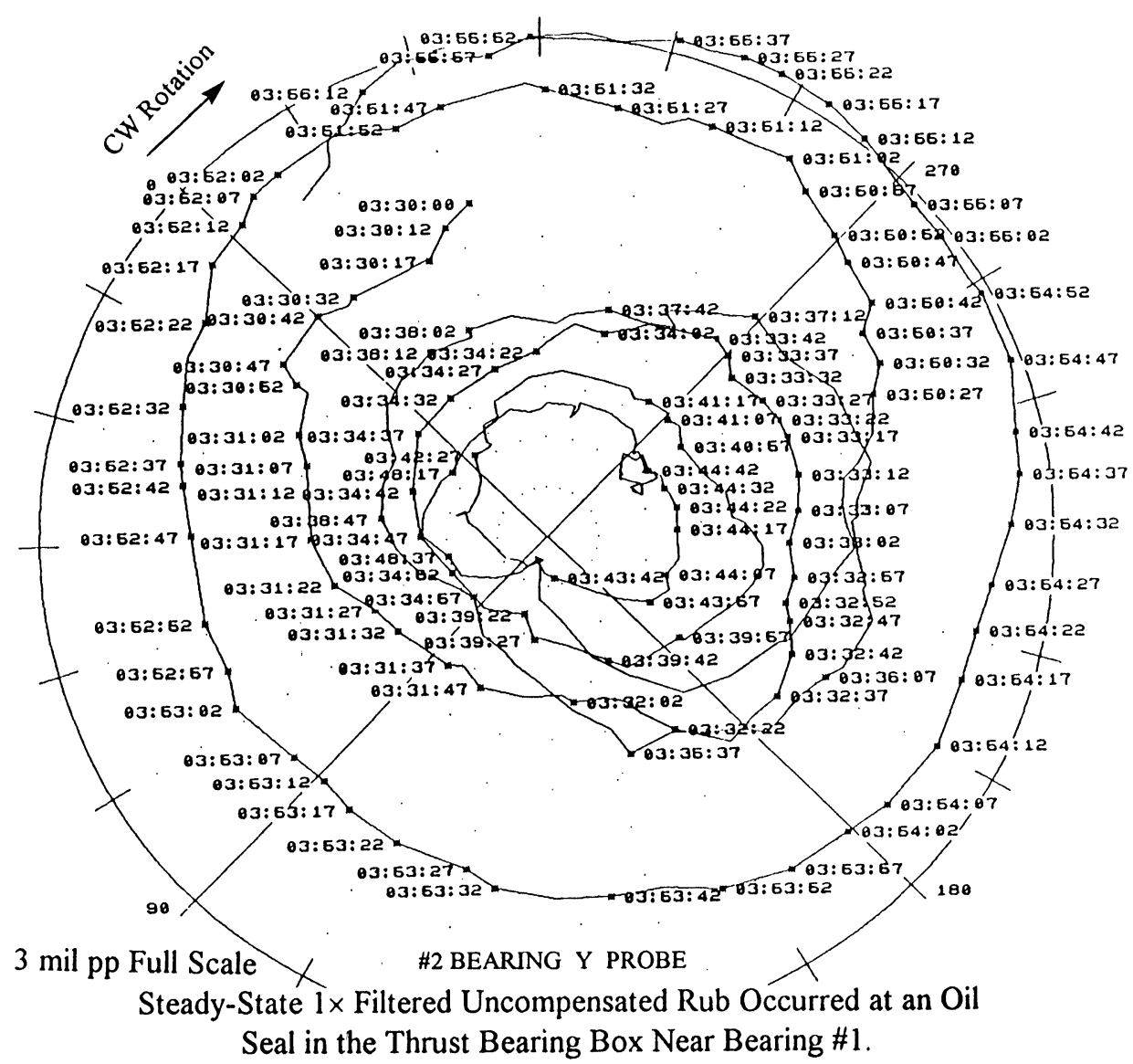

FIGURE 10 Thermal bow-related rub diagnosis: polar plot of time trended $1 \times$ response vector of a turbine rotor at $3600 \mathrm{rpm}$ during $30 \mathrm{~min}$. Vibration amplitudes vary, phase lags 6 full circles (BN MDS Reports).

$\omega_{\text {whirl }} \approx \lambda \Omega\left\{1-\frac{K_{2}^{2} D\left(1-\lambda_{R}\right)}{D_{b\left[\left(K_{1}+K_{2}-M \lambda^{2} \Omega^{2}\right)^{2}+\left[D\left(1-\lambda_{R}\right) \lambda \Omega\right]^{2}\right.}}\right\} \approx$

$\approx \lambda \Omega$ and $\omega_{\text {whip }} \approx \sqrt{\frac{K_{1}+K_{2}}{M}}$

The relationship $\omega=\omega(\Omega)$ is an inclined hyperbola; around $\Omega=(1 / \lambda) \sqrt{K_{1}+K_{2} / M}$ the values(12) are least accurate. Without the nonlinear term $\left(K_{n b}=0\right)$, Eq. (11) represents the system characteristic equation for calculation of the eigenvalues. The imaginary parts of the eigenvalues are almost the same as frequencies in Eqs. (12) (additionally, there exists the negative "whip" frequency). It has been well known in vibration theory that the self-excited vibrations exhibit frequencies close to natural frequencies of the linearized system. Small differences occur due to system nonlinearities, active at higher deflections of the elastic members. The "whip" frequency (12) asymptotically approaches the rotor lateral mode natural frequency corresponding to rotor high eccentricity operation. The "whirl" frequency (12) is purely generated by the fluid interaction and is related to the fluid film radial damping (Muszynska [1986a, b, 1988a, 1990a]). The fluid involved in the circumferential

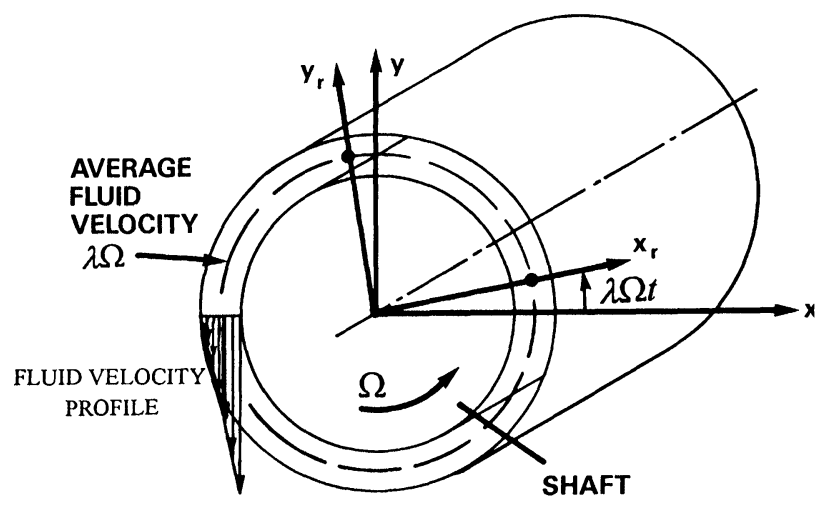

FIGURE 11 Model of a shaft rotating inside a bearing or seal. The circumferential flow-induced force rotates at angular velocity $\lambda \Omega$ (Muszynska [1988a]). 
motion transfers energy from rotation to lateral vibration. The circumferential velocity ratio $\lambda$, as a decreasing function of the rotor eccentricity, is a useful measure of this phenomenon (Muszynska [1988a]). It is also very convenient in the instability onset prediction: all thresholds of fluid-induced instabilities ${ }^{4}$ of the rotor are inversely proportional to $\lambda$. In particular, for the simple model (10) the instability onset obtained from the eigenvalues is:

$$
\Omega_{s t} \approx \frac{1}{\lambda} \sqrt{\left(K_{1}+\frac{\left(K_{b}+K_{3}\right) K_{2}}{K_{b}+K_{3}+K_{2}}\right) / M}
$$

For the rotor/seal model $\Omega_{s t}$ is close to $1 / \lambda$ times rotor lateral mode natural frequency. For the rotor/bearing model there is $K_{3}=0$, and since usually $K_{b} \ll K_{1}$, the onset (13) reduces to $\Omega_{s t} \approx \sqrt{K_{1} / M} / \lambda$. For a relatively small $K_{1}$ it may occur at quite low rotative speed, much lower than the first balance resonance speed. After this instability onset, the following limit cycle self-excited vibrations have the whirl frequency (12), and exhibit forward, almost circular, orbits (Fig. 12). If the onset (13) is high enough, only whip vibrations may be observed.

For the assumed one nonlinear term, the fluid whirl/ whip amplitude at the source location of instability is obtained from Eq. (11) as follows:

$$
\begin{aligned}
B & =\frac{1}{K_{n b}}\left\{\frac{\left(K_{1}+K_{2}-M \omega^{2}\right) K_{2}^{2}}{\left(K_{1}+K_{2}-M \omega^{2}\right)^{2}+[D(1-\lambda) \omega]^{2}}\right. \\
& \left.-K_{2}-K_{3}-K_{b}\right\}^{1 / 2}
\end{aligned}
$$

where $\omega$ are the whirl or whip frequencies which are calculated from the remaining part of Eq. (11) (Eqs. (12) present approximated values only).

For well-balanced rotors ${ }^{5}$ the whirl vibrations are quite persistent (Fig. 12). At the beginning of fluid whirl, the rotor vibrates as a rigid body. When the rotative speed approaches the $1 / \lambda$ value of the first balance resonance frequency, the rotor self-excited whirl vibrations get smoothly transformed into the whip, with a frequency

\footnotetext{
4"Instabilities" means high amplitude self-excited lateral vibrations of the rotor occurring as limit cycles of rotor unstable pure rotation.

${ }^{5} \mathrm{~A}$ higher unbalance force resulting in the rotational synchronous vibration mode moves the rotor within the clearance into higher eccentricity range, where the fluid circumferential flow is modified $(\lambda$ lowered), and the fluid film radial stiffness and damping are high. This effect is especially evident in the balance resonance range of speeds where the fluid whirl disappears (Muszynska, [1986b]). In contrast to the rub malfunction, the unbalance provides here better stability.
}

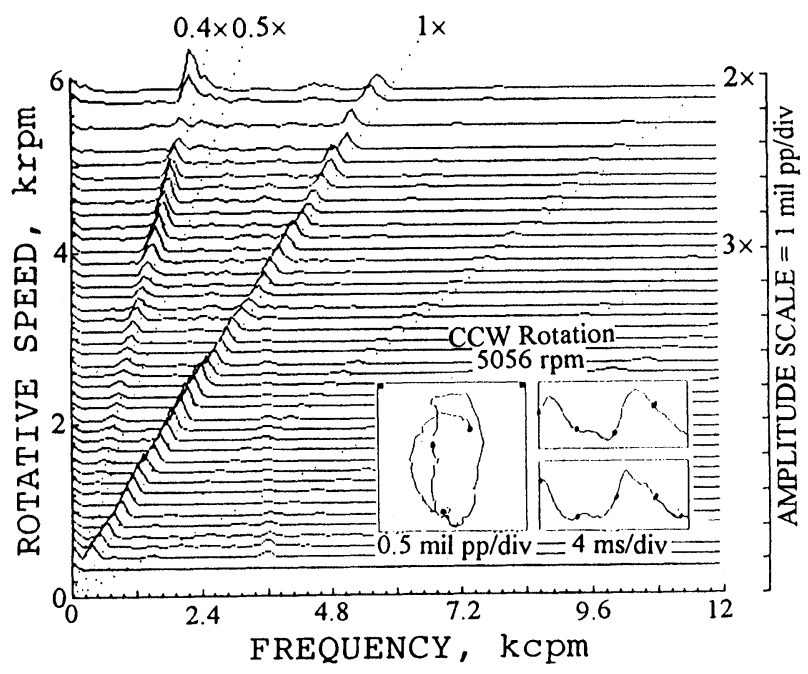

FIGURE 12 Orbit, timebase wave at $5056 \mathrm{rpm}$, and spectrum cascade of a start-up of an electric motor-driven compressor rotor supported in oil-lubricated bearings. Fluid whirl vibrations with frequency $0.4 \times$ are present (BN MDS Reports).

asymptotically tending to the rotor high eccentricity first lateral mode natural frequency (slightly modified by nonlinearities), while still maintaining forward direction, and almost circular shaft orbits at the bearing (or seal) location. The other shaft locations exhibit vibration patterns typical for the rotor first bending mode. The fluid whip is also persistent; at certain rotative speeds it may, however, disappear and then reappear again. It can be shown that there may exist cessations and subsequent onsets of the instabilities (Muszynska [1986b, 1988b]). At higher rotative speeds the consecutive onsets of instability lead to the fluid-whirl/whip vibrations of higher modes (Fig. 13) (Muszynska, [1988b, 1991]). Their frequencies and orbital appearance characteristics are similar to the first mode whirl/whip ones, but the shaft centerline exhibits distinct higher mode shapes, characteristic for these higher modes.

Even though very simplified, the mode-based model (10) indicates quite well in which direction the cures of fluid whirl/whip vibrations should go. More complex rotor systems exhibit essentially the same behavior as this simple system. The main cause of the whirl/whip vibrations lies in the fully developed circumferential flow: the rotor will become more stable if the circumferential flow-related tangential force strength is reduced $(\lambda$ lowered). This may be accomplished by either moving the shaft to higher eccentricities, by applying to the rotor a "friendly" external radial load force, or by modifying the fluid flow pattern. Appropriately loaded noncircular bearings, bearings with lobes and grooves, bearings with 


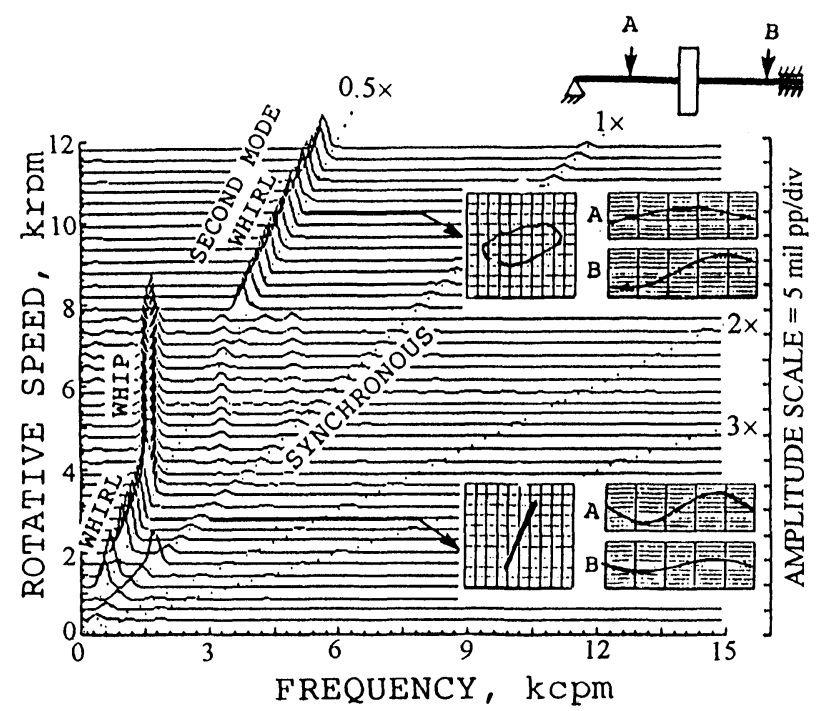

FIGURE 13 Spectrum cascade of the rotor vibrations (measured by the transducer B) exhibiting fluid whirl and whip of the first two modes, supported by measured data from two vertical transducers in the timebase and orbital modes. Shaft rotates concentrically in one brass bearing bushing the one oil-lubricated bearing (Muszynska [1988b]).

tilting pads fulfill both objectives. Seals with swirl brakes and anti-swirl injections achieve the goal of circumferential flow reduction, resulting in rotor stable operation. Shaft rotation at a higher eccentricity not only reduces $\lambda$, but also causes an increase of fluid film radial damping and stiffness. The latter is especially beneficial for the shaft stability. A larger fluid radial stiffness may also be achieved by increasing fluid pressure (externally pressurized bearings are well known for their excellent stability features). Fluid temperature changes remain inconclusive in which direction they should go in order to enhance stability. The temperature affects the fluid radial damping, as well as the tangential force (Muszynska [1988b]). Finally, better stability can be achieved by appropriately redesigning the rotor (for higher $K_{1}$ and lower $M$ the instability onset occurs at a higher rotative speed, provided that the lower mass would not affect the shaft radial load, which may result in lower shaft eccentricity).

The diagnosis of the fluid whirl/whip vibrations is relatively easy, especially when the transient start-up/ shutdown data are available. The filtered whirl/whip phase measurements allow identification of the location of instability source along the machine train (Bently et al [1990]). More difficult is, however, identification of what actually causes instability, especially when only the whip vibrations are present.

While the fluid whirl vibrations due to bearing fluid interactions have frequencies slightly lower than $1 / 2 \times$ (for most bearings they vary from $0.35 \times$ to $0.45 \times$ ), the fluid seal or stator/blade tip whirl vibrations may exhibit quite a different ratio, especially when the seals are equipped with swirl brakes or anti-swirl injections (lower $\lambda)$ or when there exists a significant level of fluid recirculation (higher $\lambda$ ), as it often happens in pumps and compressors. In the latter the whirl vibrations may even exhibit frequencies higher than the rotative frequency [(Bently Nevada MDS Reports 1987-90)].

Shaft centerline position data may indicate impending fluid-related malfunction earlier than the actual appearance of whirl/whip vibrations. A gradually decreasing eccentricity position of the journal inside the bearing clearance due to either changes in radial load forces or due to bearing surface wear warn that fluid whirl or whip may soon occur. Axial vibrations and position data may also warn about changes in flow which may lead to whirl/whip vibrations.

While rub-related vibrations are often chaotic, transient in nature, the fluid-induced vibrations are usually steady and persistent. The strength of their higher harmonics is much lower than in the rub cases. The fluid-induced whirl/whip orbits are always forward and close to circular.

\section{Loose Stationary Part Malfunction}

A looseness between the rotor-supporting pedestal and the foundation is a common malfunction in rotating machines. The unbalance force carried by the rotor may occasionally exceed the gravity force and/or other lateral forces applied to the rotor and pedestal. This causes a periodic lifting of the pedestal, resulting in system stiffness softening, its cyclic variability and impacting. As a result, similarly to the rub malfunction, the rotor may exhibit changes in the synchronous responses, and an appearance of fractional subsynchronous vibrations $(1 / 2 \times, 1 / 3 \times ; \ldots)$ in some rotative speed ranges. Most common is the occurrence of the $1 / 2 \times$ vibration component, well known in the field.

A simple model which simulates the loose pedestal malfunction includes periodic softening of the system (the equation corresponding to the direction of looseness is given only) (Goldman et al. [1991]):

$$
\begin{gathered}
M \ddot{y}+D_{R}(1-\lambda) \dot{y}+K_{R} y+v\left[D_{f} \dot{y}+K_{f}(y-c)\right. \\
\left.-K_{R} c\right]=m r \Omega^{2} \sin (\Omega t+\delta), v=\left\{\begin{array}{l}
0 \text { for }|y| \leq c \\
1 \text { for }|y|>c
\end{array}\right.
\end{gathered}
$$

where the subscripts " $f$," " $R$ " relate to foundation and rotor parameters respectively.

It has been shown that, together with local impacting effect included in the more formal analysis, this simple model generates synchronous and subsynchronous frac- 
tional frequency responses $1 / 2 \times, 1 / 3 \times, \ldots$, and represents the simplest simulation of the observed phenomena (Fig. 14) (Goldman et al, [1991]).

The diagnosis of the pedestal looseness is usually based on the appearance of $1 / 2 \times$ vibrations and visual inspection of the bearing/pedestal/foundation fastenings.

\section{Oversize, Poorly Lubricated Bearing Malfunction}

The designs of plain bearing bushings and rolling element bearings used in rotating machines include a small bearing clearance which is appropriate for normal operation of the rotor. When poorly lubricated bearing and journal surfaces wear, this inevitably leads to an increase of the rotor/bearing clearance. The increased looseness in the bearing (which is often referred to as "dead band") causes a reduction of the system stiffness, and may result in a specific array of dynamic phenomena experienced by the rotating shaft. Excessive bearing clearance, and occasional loss of the shaft/bearing contact during operation, cause variable stiffness of the rotor/bearing system, thus provide nonlinear conditions for unbalancerelated excitation, which may lead to rotor instability. The physical phenomena occurring in the oversize bearing/rotor system, namely, variable stiffness, impact-

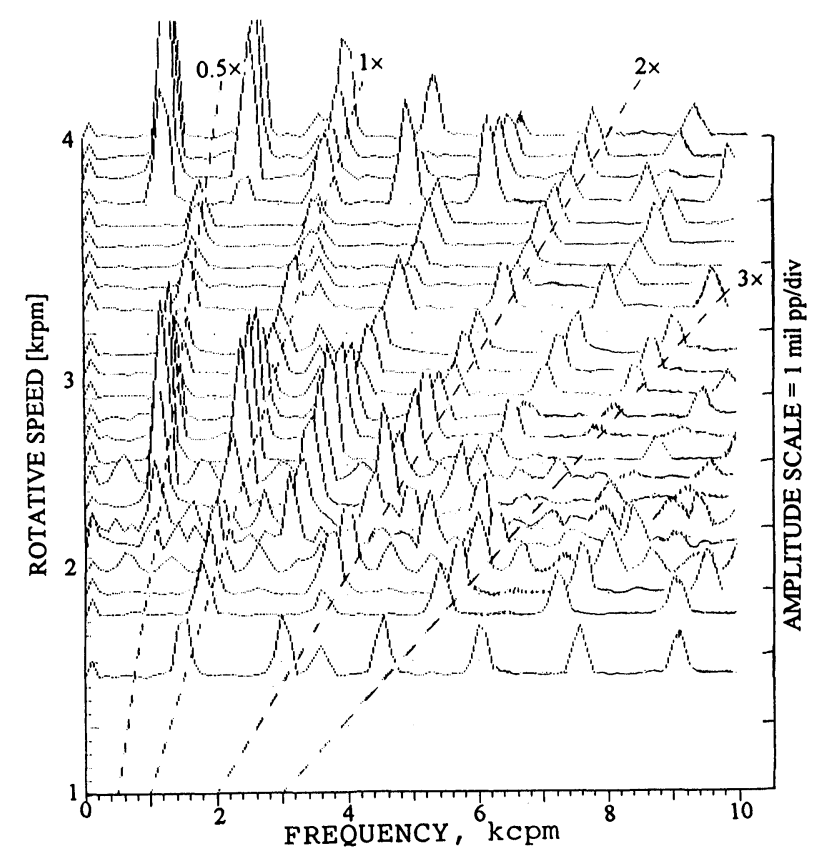

FIGURE 14 Spectrum cascade of the response of a rotor with loose pedestal. The $1 \times, 1 / 2 \times$, and $1 / 3 \times$ subsynchronous vibrations, plus rich spectrum of higher harmonics, are present (Goldman et al. [1991]). ing, and friction, are similar to the dynamic phenomena during the rotor-to-stator rubbing. The similarity is, however, of the "mirror image" type, since while rubbing, the system becomes periodically stiffer during a cycle of vibration, which leads to an increase of the average stiffness. In the oversize bearing/rotor system, the average stiffness decreases. As the rubbing occurrences are described as "normal-tight" situations, the oversize bearing leads to the "normal-loose" description. In both situations two other physical phenomena, namely friction and impacting, are similar. Their involvement and strength differ, however, in specific cases.

The mathematical model simulating the oversize bearing malfunction is similar to Eqs. (7) with $K_{3}=0$ and the normal force $N$ replacing the stator stiffness force $K_{s} \times$ $\left(\left|z_{1}\right|-c\right)$ (Muszynska [1990b]). The model produces rotor unbalance-related responses with synchronous and subsynchronous fractional frequencies, as well as selfexcited vibrations occurring with the rotor natural frequency of the first lateral mode. The perturbation equations around the rotor static equilibrium position yield anisotropic characteristics for the vertical/horizontal stiffnesses and tangential components. The latter adequately reflect the anisotropic nature of the rotor/loose bearing system, and eventually generate differences in vertical and horizontal modes.

The diagnosis of the oversize bearing malfunction, and distinguishing it from the rubbing, should be based on the rotor centerline position and $1 \times$ data, frequency spectrum, and, especially meaningful, the orbit analysis (Figs. 15, 16). While exhibiting similar spectra, the journal/bearing contact is usually maintained during a longer fraction of the vibration period when the rotor/ stator rubbing contact, thus the orbits are substantially different from the rub case. While maintaining the contact, the journal slides on the bearing surface, and a part of the orbit follows the bearing clearance circle. During rubbing there is high rotor lateral excursion from the rubbing spot, and, therefore, more impacting and unsteady transient motion occur. Within an oversize bearing the journal remains close to the bearing surface, even when the contact is broken. The consecutive contacts do not produce high power impacts, thus there are much less transient components and higher harmonics in the vibration spectrum, as compared to the rub cases.

\section{Loose Rotating Part Malfunction}

Looseness, and eventual involvement in rotative motion of machine elements, such as disks or thrust collars mounted on rotating shafts, or such as bearings untightened in bearing pedestals, represent another machine 


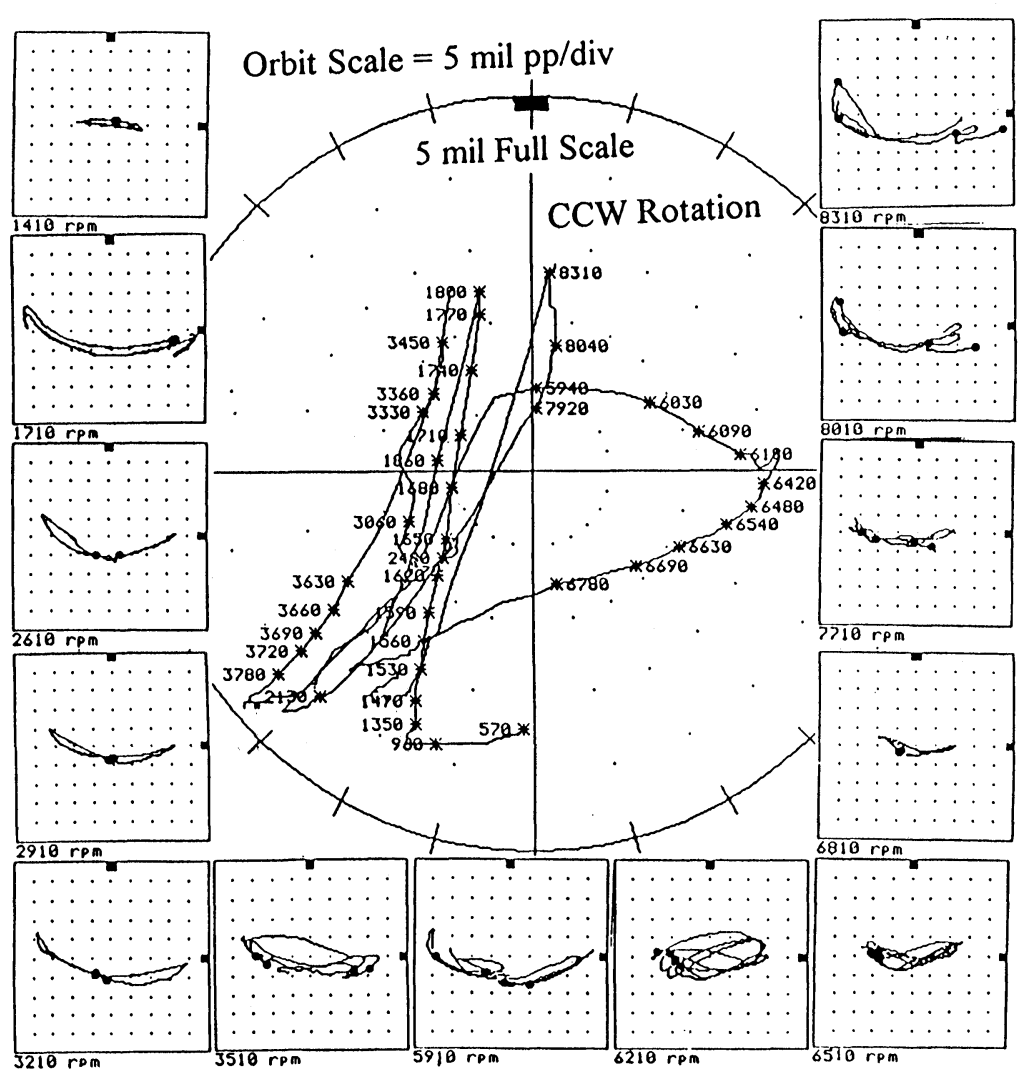

FIGURE 15 Centerline position during start-up and selected orbits of a journal rotating in an oversize poorly lubricated bearing (unbalanced rotor supported in one 5-mil and one 50-mil radial clearance brass bearing bushing) (Muszynska [1990b]).

malfunction. A disconnected disk or a thrust collar will still rotate, but at a different speed than that of the rotating shaft, and may also displace axially. A loose bearing may start rotating, dragged into rotative motion by rotating shaft.

The clearances, friction conditions between the shaft and the loose part, as well as the tangential external force applied to the loose part (such as external fluid dynamic drag) play important roles in the rotor dynamic response. While perturbing the normal operation of the machine, this type of looseness-related dynamic phenomena can, however, be relatively easy to identify and eventually corrected, as it causes very characteristic modifications of rotor normal operational responses. A loose rotating part usually carries an unbalance which changes the balance state of the machine. This results in a modification of the synchronous $1 \times$ vibrations, and in an appearance of the loose part unbalance-related forced component.

A simple model describes this phenomenon (an axial displacement of the loose part is not included) (Muszynska [1988c]):

$$
\begin{aligned}
& M \ddot{z}+D(1-\lambda) \dot{z}+K z= \\
& =m_{R} r_{R} \Omega^{2} e^{j\left(\Omega t+\delta_{R}\right)}-m_{\ell} c \omega_{\ell}^{2} e^{j \omega_{\ell} t}, \\
& \omega_{\ell}=\omega_{\ell}(c, \mu, \vartheta)
\end{aligned}
$$

where the subscripts " $R$ " and " $\ell$ " refer to the rotor and loose rotating part respectively. The loose part rotative frequency $\omega_{\ell}$ is a function of the shaft/loose part clearance, surface friction, and the tangential drag coefficient $\vartheta$, provided by the fluid environment. Depending on a particular machine, the latter can drive the loose part at higher frequency than $\Omega$ (e.g., a loose turbine disk) or slow down the loose part, relatively to the rotor. In both situations $\mu$ and $\vartheta$ act in opposite directions. At steadystate conditions the friction and fluid drag may balance each other, and $\omega_{\ell}$ becomes constant. If it does not differ very much from the rotative speed, the resulting vibrations exhibit the characteristic pattern of beat (Fig. 17). Most often, however, the looseness of a rotating part leads to transient conditions. The loose-part-related vibrations have most often a subsynchronous frequency tending to the natural frequency of the rotor. These vibrations look somewhat similar to fluid whirl/whip 


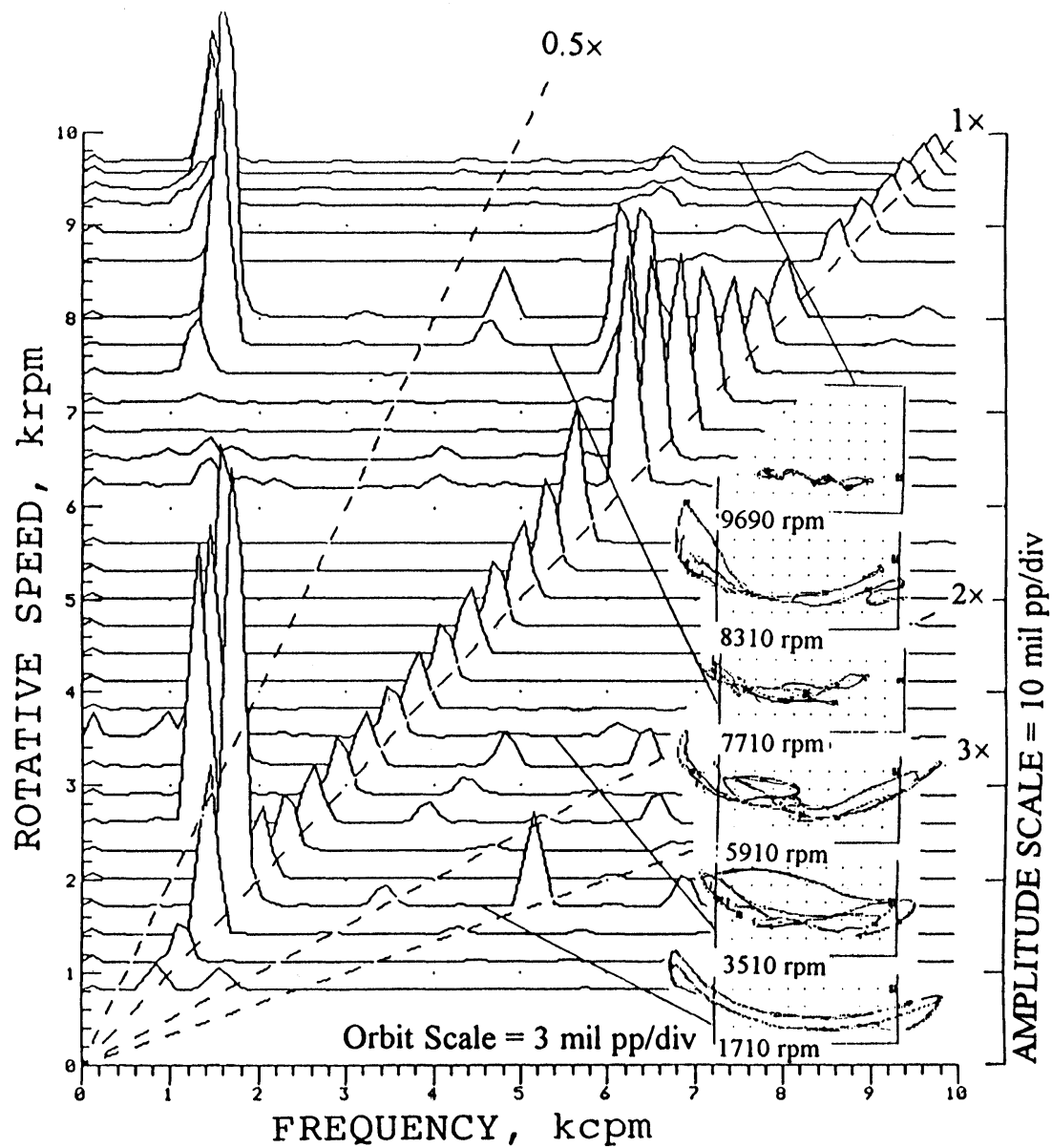

FIGURE 16 Spectrum cascade of the journal horizontal response and selected orbits from the same run as in Figure 15. Subsynchronous vibrations of $1 / 2 \times$ and $1 / 3 \times$, as well as self-excited vibrations, are present (Muszynska [1990b]).

vibrations, and may sometimes be confused with the latter (Fig. 18).

\section{Cracked Shafts}

Cracked shafts represent a severe malfunction in rotating machinery, and always should be considered as a potential event. A shaft crack causes two major results: a stiffness reduction resulting in rotor anisotropy, and a shift of the elasticity axis (the rotor bows) which affects the balance state.

In order to present a simple model of a rotor with the cracked shaft, first transform Eq. (1) of an unbalanced rotor into coordinates $z_{r}=x_{r}+j y_{r}=z e^{-j \Omega t}$ attached to the rotor, and rotating at the angular speed $\Omega$ (assuming further that the axis $y_{r}$ passes through the crack):

$$
\begin{aligned}
& M\left(\ddot{z}_{r}+2 j \Omega \dot{z}_{r}-\Omega^{2} z_{r}\right)+D(1-\lambda)\left(\dot{z}_{r}+j \Omega z_{r}\right)+ \\
& K z_{r}-j K_{t} z_{r}=m r \Omega^{2} e^{j \delta}+P e^{j(-\Omega t+\gamma)}
\end{aligned}
$$

The model of the cracked rotor will be as follows (Muszynska, 1982):

$$
\begin{aligned}
& M\left(\ddot{z}_{r}+2 j \Omega \dot{z}_{r}-\Omega^{2} z_{r}\right)+D(1-\lambda)\left(\dot{z}_{r}+j \Omega z_{r}\right)+ \\
& K z_{r}-j K_{t} z_{r}-j K \Delta y_{r}=m r \Omega^{2} e^{j \delta}+P e^{j(-\Omega t+\gamma)}-j K \epsilon
\end{aligned}
$$

where $0<\Delta<1$ represents the gaping crack-related, unilateral stiffness-reducing effect and $\epsilon$ represents the rotor elasticity axis shift. When Eq. (18) is re-transformed to the previously used stationary coordinates, it will have periodically variable stiffness with frequency $2 \Omega$, and an additional unbalance-like excitation $K \epsilon e^{j\left(\Omega t+270^{\circ}\right)}$. Since the latter interferes with the rotor residual unbalance " $m r$," the synchronous $1 \times$ responses of a cracked rotor will differ from the original ones, especially at the low frequency range. The radial load force $P$ applied to a rotor with $2 \times$ periodically variable stiffness results in an appearance of the forced vibrations with frequency $2 \times$. (Eq. (18) can easily be solved first, 

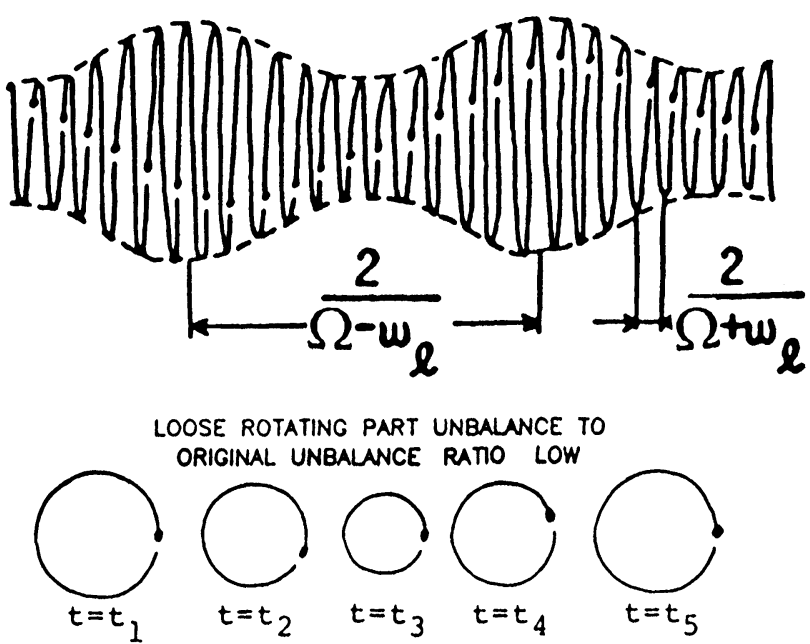

LOOSE ROTATING PART UNBALANCE TO ORIGINAL UNBALANCE RATO LOW
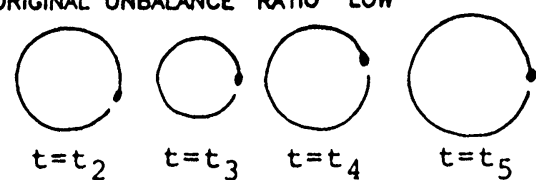

AMPUTUDE VARIATION SMALL KEYPHASOR EXCURSION SMALL

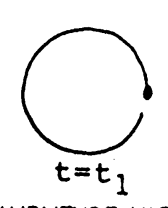

HIGH RATIO OF UNBALANCES
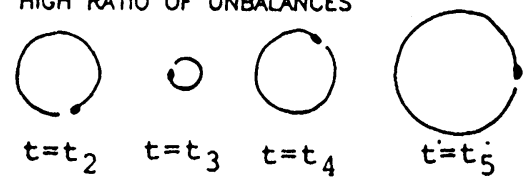

AMPLITUDE VARIATION LARGE

KEYPHASOR EXCURSION LARGE (FULL $360^{\circ}$ )

FIGURE 17 Loose rotating part at a steady-state machine operation conditions causes beat vibrations due to unbalance rotating at frequency slightly lower than the rotative speed (Muszynska [1988c]). Timebase waveform and orbits.

then transformed to stationary coordinates to show the solution comprising modified synchronous and double frequency responses). The crack-related increased stiffness differences in two orthogonal directions of the rotor cross section, modeled through the coefficient $\Delta$, cause actually an appearance of another tangential force acting

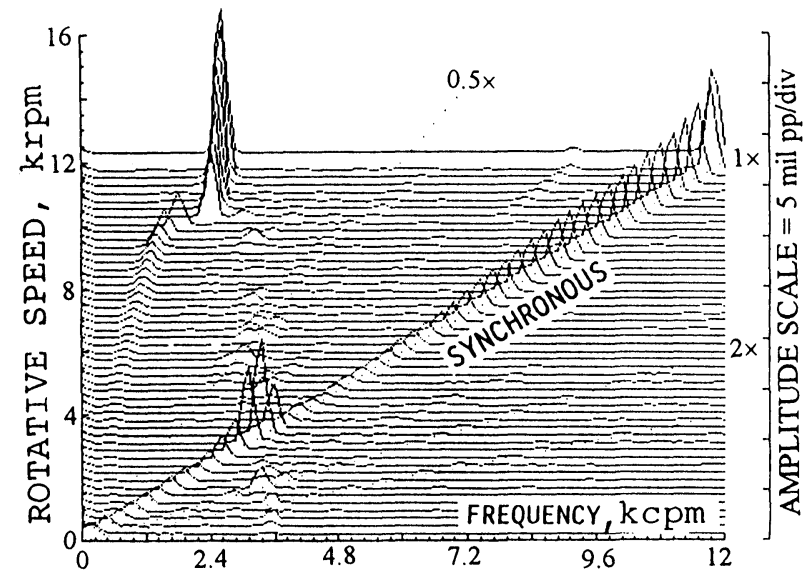

FIGURE 18 Spectrum cascade of vibration response during start-up of a rotor with a loose rotating disk. The frequencies of the rotative speed dependent subsynchronous vibrations tend to the natural frequency of the rotor bending mode (Muszynska [1988c]). in the direction of rotation (another mechanism of transferring rotating energy into lateral vibrations). This tangential force opposes damping, thus results in a decrease of the system effective damping. The model (18) predicts that if $\Delta$ reaches four times synchronous damping factor $(\Delta=4 \times D /(2 \sqrt{K M}))$, then the effective damping is nullified. Such value of $\Delta$ can practically never be achieved before a total destruction of the machine. A gradual decrease of the effective damping can, however, easily be noticed earlier in $1 \times$ and $2 \times$ resonant amplitude increases.

The early diagnosis of shaft cracks is based mainly on two symptoms: changes in $1 \times$ and/or $2 \times$ vibration vectors (Bently et al [1986]). These data can be monitored at steady-state, as well as during transient start-up/ shutdown conditions (Figs. 19, 20). The latter data is especially meaningful, as the vibration changes are particularly significant in the $1 \times$ and $2 \times$ lateral mode resonant ranges of rotative speeds. Deviation of $1 \times$ and $2 \times$ vibration vectors from their acceptance regions (established based on normal operating condition including radial load information) provides alarming and early warning of a cracked rotor. The rotor centerline position and slow roll data give additional information about the rotor bow situation. Observation of the orbits is also very helpful in diagnosing the shaft crack. A high $2 \times$ component in the $2 \times$ resonance range of speeds causes an appearance of an internal loop on the orbit $(2 \times$ orbit is forward).

The crack-related weakening of the rotor in a constant operation may be diagnosed by analyzing the $1 \times$ and $2 \times$ vector trend data. A shift in the natural frequency can be detected when at a constant rotative speed, at certain moment of time, the $1 \times$ or $2 \times$ vectors exhibit resonant features (Fig. 21). With an assumed rate of crack propagation, the timing of the total shaft breakage can be estimated.

\section{Torsional/Lateral Coupled Vibrations}

In most machines the torsional natural frequencies are in much higher range than those of lateral modes. Typical forcing functions existing in the rotating machines (except those which contain gear transmissions and those with variable speed drivers) do not excite high torsional vibrations. The situation changes if the torsional natural frequencies are lower, and within the range of the operating speeds. Shaft asymmetry, radial load, and unbalance which are affecting the position of bending and twisting centerlines constitute the coupling factors between torsional and lateral modes.

A simple asymmetric rotor model which takes into consideration the lateral and torsional coupling is as 


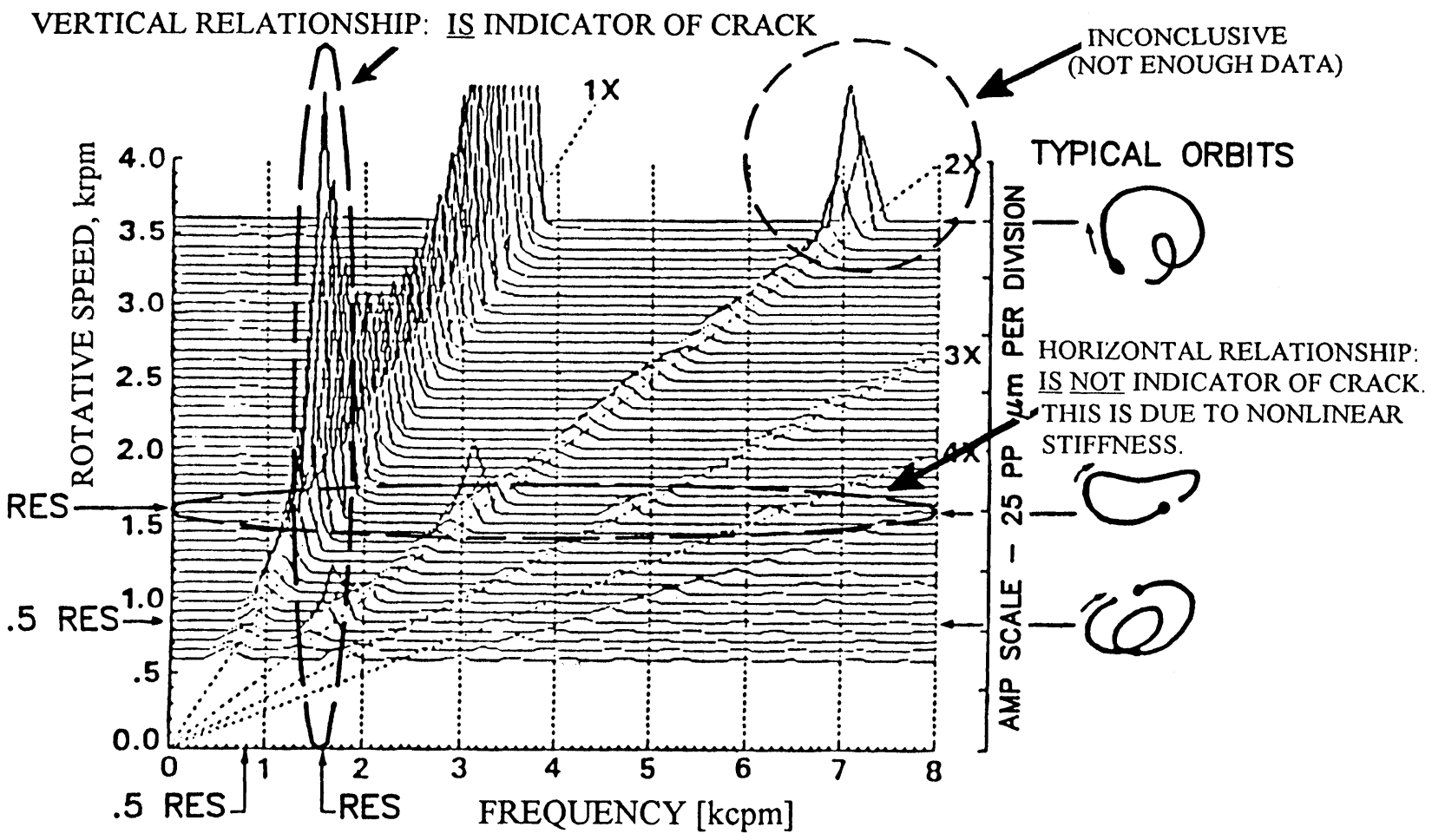

FIGURE 19 Spectrum cascade and selected orbits of a cracked shaft vibrational response. High $1 \times$ and $2 \times$ components. The latter exhibit resonances at "half resonance" speeds of (Bently et al. [1986]).

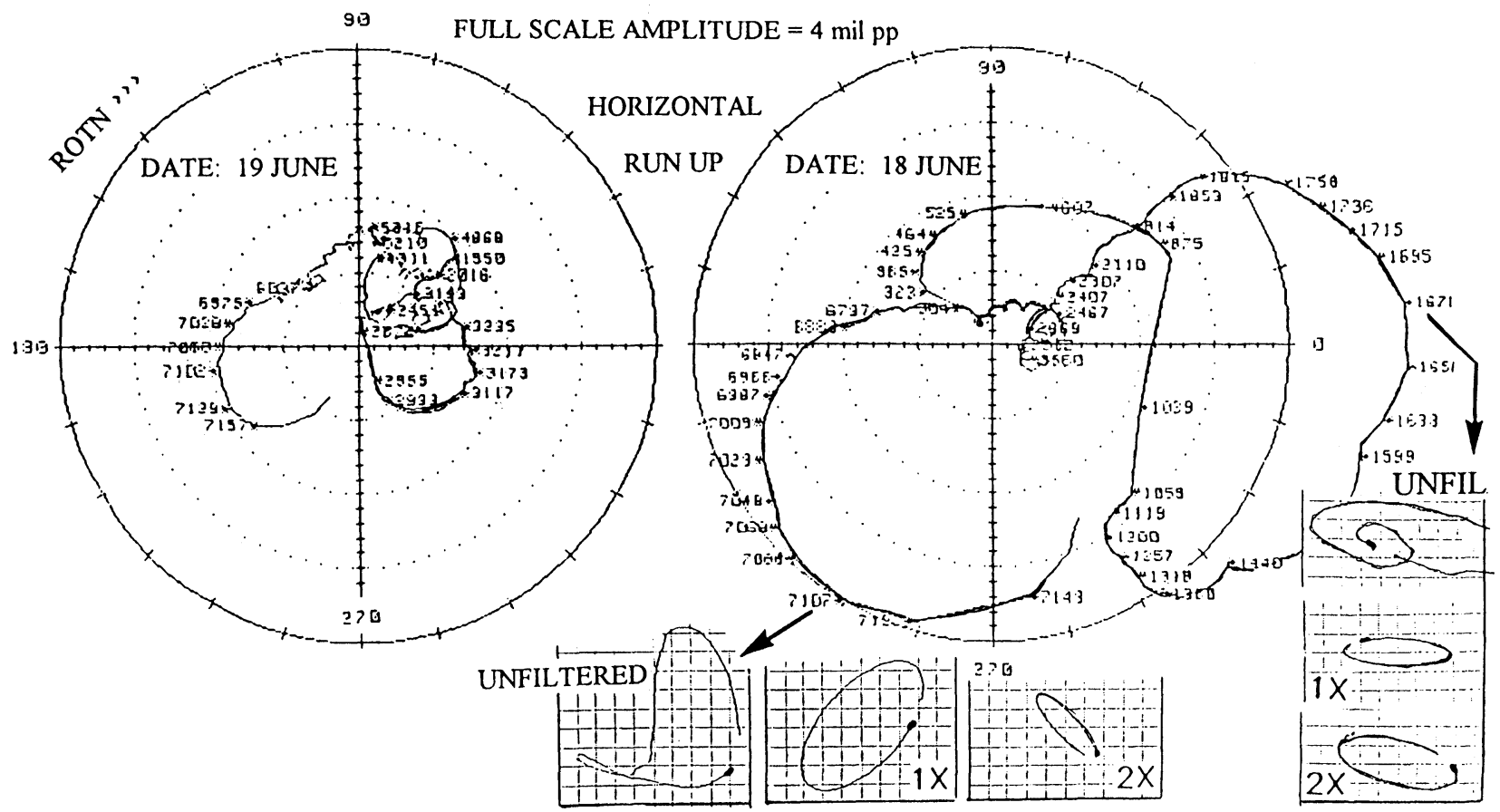

FIGURE 20 Shaft crack diagnosis: abnormal $2 \times$ response vector polar plot taken 9 days after the first one. Amplitudes considerably increased, and phase is leading in the low speed range. At $3550 \mathrm{rpm} 2 \times$ orbit is backward (BN MDS Reports). 
follow (Loewy et al., 1969):

$$
\begin{aligned}
& M\left(\ddot{z}_{r}+2 j \Omega \dot{z}_{r}-\Omega^{2} z_{r}\right)+D(1-\lambda)\left(\dot{z}_{r}+j \Omega z_{r}\right)+ \\
& K z_{r}-j K_{\tau} z_{r}-j K \Delta y_{r}-m r\left[2 \omega \dot{\psi}+j\left(\psi \Omega^{2}-\ddot{\psi}\right)\right] e^{j \delta}=(19) \\
& m r \Omega^{2} e^{j \delta}+P e^{j(-\Omega t+\gamma)} \\
& I \ddot{\psi}+K_{t} \psi-\frac{m r}{M}\left[K(1-\Delta) y_{r}+D(1-\lambda)\left(\dot{y}_{r}-\Omega x_{r}\right)-\right. \\
& P \psi \cos (\Omega t+\delta-\gamma)-P \sin (\Omega t+\delta-\gamma)]=T
\end{aligned}
$$

A part of Eq. (19) is the same as (18) (with no additional crack-related unbalance); it also includes the coupling terms with the torsional vibration coordinate $\psi$. Eq. (20) represents the balance of moments for the shaft torsional mode, including the external torque $T$. This linearized equation has one periodically variable coefficient. Its action affects the torsional stiffness $K_{t}$. It is well known that such equation produces parametric resonances (Mathieu/Hill/Duffing's type). These resonances occur at rotative speeds coinciding with $1,1 / 2,1 / 4,1 / 6,1 / 8$, fractions of the torsional natural frequency $\left(\approx \sqrt{K_{t} / l}\right)$. Since the unbalance and radial load force interfering with shaft asymmetry constitute the actual system forcing functions, the highest torsional mode resonances occur at $1 \times$ and $2 \times$ (Fig. 22) (Muszynska et al. [1992]). The lateral modes exhibit peak responses at the lateral balance resonance frequencies, as well as at the speeds corresponding to the torsional natural frequency (Fig. 23). It can be noticed that while lateral mode peaks are not very high, the torsional resonant vibrations exhibit significant amplitudes, indicating, that the effective damping in the torsional mode is very poor.
A lack of recommendations to routinely install torsional vibration transducers on rotating machinery in order to monitor rotor torsional activities may prove to be a handicap: poorly damped torsional modes excited through coupling with the lateral modes contribute considerably to shaft stress increase, and may significantly intensify shaft cracking. When trended, the torsional vibration changes may reveal symptoms of an impending crack at much earlier levels than lateral vibrations.

\section{Internal/Structural Friction. Interactive Malfunctions. Extended Models}

One classical machinery rotor malfunction, namely internal and structural friction, has not yet been mentioned. Internal/structural friction in rotating elements is known to cause self-excited lateral vibrations with frequencies close to rotor natural frequencies (the model (10) with $\lambda=1$ and $D_{b}$ equal to the internal/structural friction damping may be used as a first approach in modeling these phenomena). Since internal/structural friction-induced, self-excited vibration exhibit the whip type (as the onset of stability is high enough with $\lambda=1$ ), it is usually difficult to identify a real cause of these vibrations. (Most often the whip is caused by fluid interactions, as much stronger agents.) One aspect of the internal/structural friction should, however, be mentioned here: The internal friction force acts in phase with the external damping, i.e., it intensifies the effective damping only for super synchronous frequencies. For subsynchronous frequencies, it opposes the external damping, reducing its stabilizing effect. That is why all subsynchronous vibra-

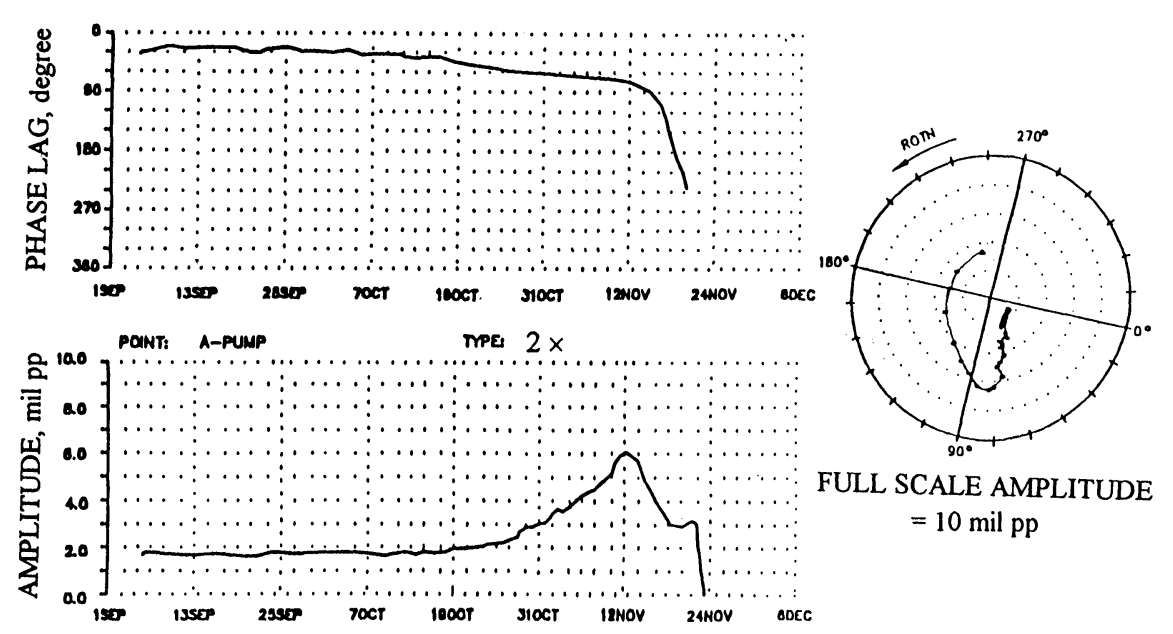

FIGURE 21 Shaft crack diagnosis: amplitude-phase-time (APHT) plot and polar trend of a cracking rotor $2 \times$ vibration component, at a constant speed. The plots exhibit the characteristics of the lateral second mode resonance response, due to the propagating crack-related shaft weakening (BN MDS Reports). 


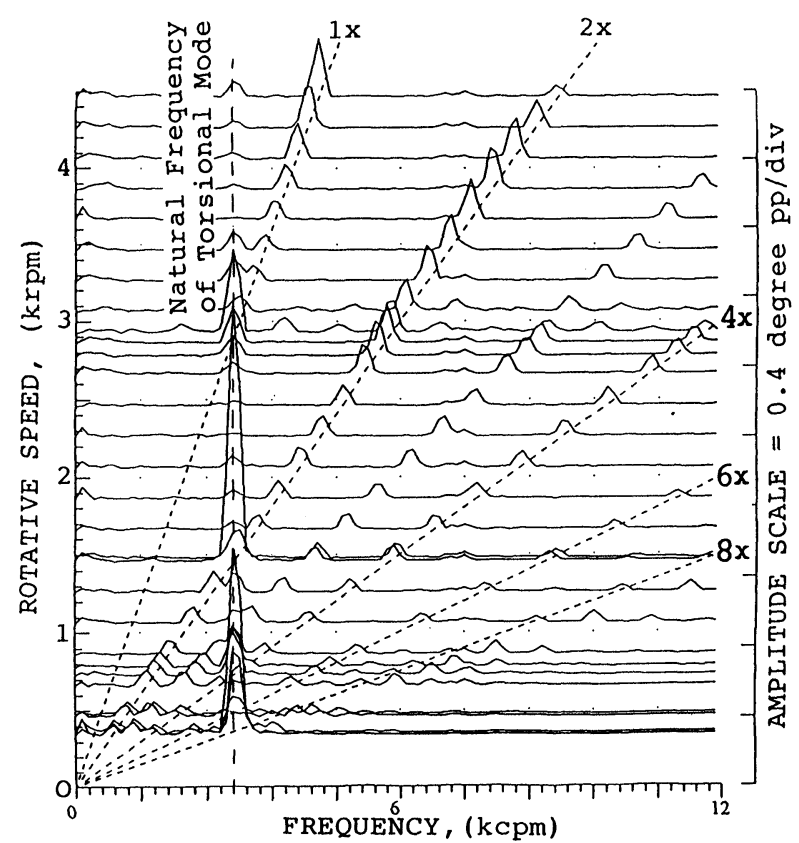

FIGURE 22 Spectrum cascade of an asymmetric rotor torsional response during start-up. The highest peak of $2 \times$ component occurs at a half torsional natural frequency speed. The peak responses for the torsional natural frequency also appear at $8 \times, 6 \times, 4 \times$, and $1 \times$. The $1 \times, 2 \times$ components, and higher harmonics of torsional vibrations due to coupling with lateral modes, are present in the entire speed range (Muszynska et al. [1992])

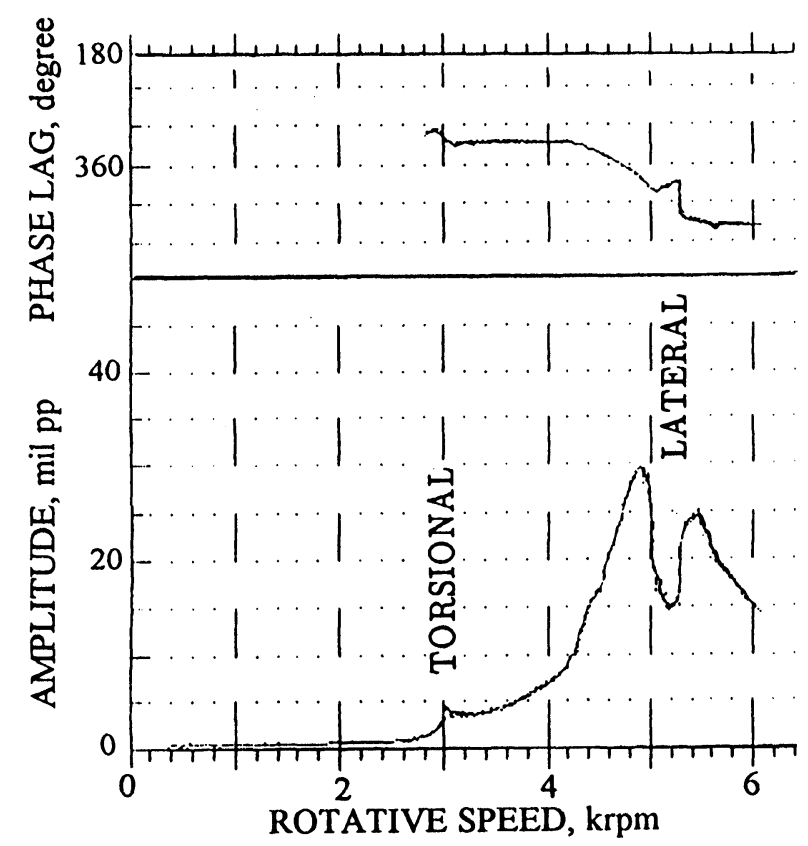

FIGURE 23 Bode plot of $1 \times$ the rotor lateral response from the same run as in Figure 22. It exhibits lateral split resonance peaks at $4900 \mathrm{rpm}$ and $5400 \mathrm{rpm}$, as well as the torsional resonance peak at $3000 \mathrm{rpm}$ (Muszynska et al. [1992]). tions caused by any other mechanism may exhibit very high amplitudes. Even though the internal/structural friction does not act as a primary cause of the rotor instability, it certainly promotes it and intensifies its appearance.

The most often occurring rotating machine malfunctions were modeled here using simple lowest mode models, emphasizing physical principles of the "cause and effect" relationships. The dynamic behavior of a rotating machine is certainly more complex, and the models adequately describing the machine dynamic process should appropriately be extended. The first basic simplification used in several models presented above concerned the rotor/bearing/pedestal structure isotropy. In machinery this almost never takes place, and the first and higher lateral modes have usually split lateral characteristics, thus the models must take into consideration the anisotropy of the system. Adequate models should cover an appropriate number of modes. The modes should include gyroscopic effects and other coupling mechanisms. Dynamically variable driving and load torques should be explicitly present in the vibrational mode relationships. The latter should also include all possible malfunction effects in interactive manners.

\section{ADVANCEMENT TREND IN VIBRATION MONITORING AND DIAGNOSTICS OF ROTATING MACHINERY HEALTH}

The analysis of accumulated database from machinery monitoring systems, and from laboratory simulations, allows for generalizations and correlations of particular malfunctions with the machine design features and operational conditions. Machinery health monitoring alone will not improve machine performance. The accumulated practical knowledge supported by simple mathematical modeling must be absorbed and implemented by machinery manufacturers (Diewald et al. [1990]). Potential mechanisms of energy transfer from rotation to vibrations should be eliminated, or at least reduced, at the design stage by implementing passive and active methods of vibration control. Machine malfunctions should not occur, as a rule, but as extraordinary events. This does not lead, however, to a conclusion that vibration monitoring systems on machinery should be abandoned. To the contrary, they would allow continuous monitoring of the machine performance, and use of the information for more efficient scheduling of routine outages.

Nowadays health monitoring of machines becomes increasingly automated. The use of computer-controlled 
instruments and computer systems to collect, record, and process the data has become a routine. Expert systems are natural extensions of this trend. About ten years ago, aspirations in the area of expert systems were being discussed. Today attempts of their designs are being published (Lu et al. [1986], Gross et al. [1989]). Several years from now, accomplishments will be summarized, followed by their routine implementations.

Contemporary expert systems are cost effective only when applied to specific classes of machines which operate in large numbers. High complexity and resulting high development costs of the expert systems are realistic only if they can be amortized over a large number of sales. The individual specific solutions for expert systems are necessary, because the general solution for the vibration pattern recognition problem for a wide variety of rotating machines operating in a large multitude of processes is still imperfect at the present time. A second major problem with contemporary expert systems is that the responses and recommendations are stated only as probabilities, thus expert human judgments are still required to assess these probabilities. In a few years, expert systems will evolve from tools that assist experts into tools that will be able to help knowledgeable users who are not experts. Only in the distant future will expert systems be able to help general users. (Advances in the neural network computing and fuzzy logic may speed up, however, this process.)

A true expert system must be capable of totally replacing a human expert in the machine operation control, diagnosis, and corrective actions. In the machinery diagnostics field, a true expert system would have to be able to recognize early signs of any potential problem by analyzing real-time data from the vibration monitoring and process control systems, hypothesize all possible causes of the problem, construct a series of parametric or process perturbation tests, and develop a data acquisition strategy to gather the required additional information to prove or disprove each hypothesis, conduct the tests, gather the data, analyze the results with respect to the particular machinery, process them, form a reliable conclusion, and undertake corrective actions. An expert system capable of performing all of these functions should be able to handle most typical problems with well-behaved symptoms on traditional machinery. $\mathrm{Hu}-$ man experts, with their intuitive abilities, will probably always be required to provide solutions for most infrequently encountered problems, or almost all malfunctions which exhibit any asymptomatical characteristics.

Expert systems must always be considered, however, as useful tools to assist in the machinery health diagnosis, not as main goals. The latter is still the safe, efficient, cost-effective, and reliable operation of the machinery used to produce required products.

\section{References}

Bently, D. E., and Muszynska, A., 1986. Detection of Rotor Cracks, Proceedings of the Texas A\&M University 15th Turbomachinery Symposium and Short Courses, Corpus Christi, Texas, pp. 129-139. Bently, D. E., and Bosmans, R. F., 1990. A Method to Locate the Source of a Rotor Fluid-Induced Instability Along the Rotor, The Third International Symposium on Transport Phenomena and Dynamics of Rotating Machinery, Honolulu, Hawaii.

Bently Nevada Mechanical Diagnostic Services Reports, 1987-1990. Diewald, W., and Nordmann, R., 1990. Parameter Optimization for the Dynamics of Rotating Machinery, Proceedings of the Third IFToMM International Conference on Rotordynamics, Lyon, France.

Goldman, P., and Muszynska, A., 1991. Analytical and Experimental Simulation of Loose Pedestal Dynamic Effects on a Rotating Machine Vibrational Response, Rotating Machinery Dynamics, DE-vol. 35, The Thirteenth Biennial ASME Conference on Mechanical Vibration and Noise, Miami, Florida.

Gross, K. C., and Humenik, K. E., 1989. Nuclear Plant Component Surveillance Implemented in SAS Software, SAS Users Group International Conference, San Francisco.

Laws, W. C., and Muszynska, A., 1987. Periodic and Continuous Vibration Monitoring for Preventive/Predictive Maintenance of Rotating Machinery, Trans. of the ASME, Journal of Engineering for Gas Turbines and Power, vol. 109, pp. 159-167.

Loewy, R. G., and Piarulli, V. J., 1969. Dynamics of Rotating Shafts, The Shock and Vibration Information Center.

Lu, S. C-Y, and Komanduri, R. (Eds.), 1986. Knowledge-Based Expert Systems for Manufacturing, ASME Publication PED-v. 24, H00371. Muszynska, A., 1982. Shaft Crack Detection, Proc. of the Seventh Machinery Dynamics Seminar, National Research Council, Edmonton, Alberta, Canada.

Muszynska, A., 1984a. Partial Lateral Rotor-to-Stator Rubs, The Third International Conference on Vibrations in Rotating Machinery, IMechE, C 281/84, York, New United Kingdom, pp. 227-236.

Muszynska, A., 1984b. Synchronous and Self-Excited Rotor Vibrations Caused by a Full Annular Rub, Proceedings of the Eighth Machinery Dynamics Seminar, NRC No. 23619, Halifax, Nova Scotia, Canada, pp. 22.1-22.21.

Muszynska, A., 1986a. Modal Testing of Rotor/Bearing Systems, International Journal of Analytical and Experimental Modal Analysis, vol. 1, no. 3, pp. 15-34.

Muszynska, A., 1986b. Whirl and Whip-Rotor/Bearing Stability Problems, Journal of Sound and Vibration, vol. 110, no. 3, pp. 443-462.

Muszynska, A., 1988a. Improvements in Lightly Loaded Rotor/Bearing and Rotor/Seal Models, Trans. of the ASME, Journal of Vibration, Acoustics, Stress and Reliability in Design, vol. 110, no. 2, pp. 129-136.

Muszynska, A., 1988b. Multi-Mode Whirl and Whip in Rotor/Bearing Systems, Dynamics of Rotating Machinery, The Second International Symposium on Transport Phenomena, Dynamics, and Design of Rotating Machinery, vol. 2, Hemisphere Publishing Corp., Honolulu, Hawaii, pp. 326-340.

Muszynska, A., 1988c. Effects of Loose Rotating Parts on Rotor Dynamics, The Fourth International Conference on Vibrations in Rotating Machinery, IMechE, C241/88, Edinburgh, Scotland, pp. 573-578. 
Muszynska, A., Bently, D. E., Franklin, W. D., and Hayashida, R. D., 1989a. Identification of Modal Parameters of Rotating Systems Using Perturbation Techniques, Part 1 and Part 2, Rotating Machinery Dynamics, DE-V. 18-1 H0508A, Twelfth Biennial ASME Conference on Mechanical Vibration and Noise, Montreal, Canada, pp. 107-118. Muszynska, A., 1989b. Rotor-to-Stationary Element Rub-Related Vibration Phenomena in Rotating Machinery, Literature Survey, The Shock and Vibration Digest, vol. 21, no. 3, pp. 3-11.

Muszynska, A., and Bently, D. E., 1990a. Frequency Swept Rotating Input Perturbation Techniques and Identification of the Fluid Force Models in Rotor/Bearing/Seal Systems and Fluid Handling Machines, Journal of Sound and Vibration, vol. 143, no. 1, pp. 103-124.

Muszynska, A., 1990b. Effect of an Oversize Poorly Lubricated Bearing on Rotor Dynamic Response, ISROMAC-4, Honolulu, Hawaii, pp. 432-452.

Muszynska, A., and Grant, J. W., 1991. Stability and Instability of a Two-Mode Rotor Supported by Two Fluid-Lubricated Bearings, Trans. of the ASME, Journal of Vibration and Acoustics, no. 2.

Muszynska, A., Goldman, P., and Bently, D. E., 1992. Torsional/Lateral Cross Coupled Responses Due to Shaft Anisotropy: A New Tool in Shaft Crack Detection, Vibrations in Rotating Machinery, IMechE, C 432-090, Bath, United Kingdom.

\begin{tabular}{|c|c|}
\hline \multicolumn{2}{|c|}{ Nomenclature } \\
\hline$A, \alpha$ & $\begin{array}{l}\text { Amplitude and phase of rotor filtered vibration } \\
\text { components }\end{array}$ \\
\hline$B, B_{1} \beta$ & $\begin{array}{l}\text { Amplitudes and relative phase of rotor self- } \\
\text { excited vibrations }\end{array}$ \\
\hline$c$ & Radial clearance \\
\hline$D, K, M$ & $\begin{array}{l}\text { Rotor modal damping, stiffness and mass } \\
\text { respectively ( } K \text { may include bearing stiffness.) }\end{array}$ \\
\hline \multicolumn{2}{|c|}{$e=2.718 \ldots$} \\
\hline$F, \delta_{I}$ & $\begin{array}{l}\text { Amplitude and angular orientation of } \\
\text { externally applied perturbation force }\end{array}$ \\
\hline \multicolumn{2}{|c|}{$j=\sqrt{-1}$} \\
\hline$I$ & Rotor polar moment of inertia \\
\hline$K_{\tau}$ & Tangential force coefficient \\
\hline$m, r, \delta$ & $\begin{array}{l}\text { Mass, radius, and angular position of } \\
\text { unbalance }\end{array}$ \\
\hline$N$ & Shaft/bearing normal force \\
\hline$P, \gamma$ & $\begin{array}{l}\text { Radial force amplitude and its angular } \\
\text { crientation }\end{array}$ \\
\hline$t$ & Time \\
\hline$T$ & Torque \\
\hline$x, y$ & $\begin{array}{l}\text { Rotor lateral displacements in two orthogonal } \\
\text { directions (conventionally } x=\text { horizontal, } y= \\
\text { vertical) }\end{array}$ \\
\hline$z, z_{1}$ & Rotor lateral displacement coordinates \\
\hline$\Delta, \epsilon$ & $\begin{array}{l}\text { Crack-related stiffness-reducing coefficient and } \\
\text { elasticity axis shift respectively }\end{array}$ \\
\hline$\vartheta$ & Fluid-related tangential drag coefficient \\
\hline$\lambda$ & $\begin{array}{l}\text { Fluid circumferential average velocity ratio, a } \\
\text { measure of the strength of circumferential flow } \\
\text { in bearings, seals, and/or rotor/stator periphery }\end{array}$ \\
\hline$\mu$ & Dry friction coefficient \\
\hline$v$ & Kronecker's delta \\
\hline$\psi$ & Torsional angle coordinate \\
\hline$\omega$ & $\begin{array}{l}\text { Perturbation frequency, frequency or } \\
\text { self-excited vibrations, rotor precession } \\
\text { frequency }\end{array}$ \\
\hline$\Omega$ & Rotative speed \\
\hline
\end{tabular}

$\begin{array}{ll}\text { Subscripts } & \\ b & \text { Bearing, seal, or rotor/stator periphery fluid } \\ f & \text { film } \\ \ell & \text { Foundation } \\ n & \text { Loose rotative part } \\ r & \text { Nonlinear } \\ R & \text { Rotating coordinate } \\ s & \text { Rotor } \\ s t & \text { Stator } \\ t, x, y & \text { Stability } \\ \tau & \text { Torsional, horizontal, vertical }\end{array}$

\section{APPENDIX}

\section{Vibration Transducers}

Three types of transducers are available for measuring mechanical vibrations.

1. Displacement transducer. Most reliable and useful transducer to measure rotor vibrations relative to stationary elements of the machine is a noncontacting eddy current proximity probe (Fig. A1). Its principle of operation is based on a modification of electromagnetic field, due to currents induced in a conductive solid material in the proximity of the probe tip. The output voltage is proportional to the gap between the probe and the material surface. Typical sensitivity is $0.2 \mathrm{~V} / \mathrm{mil}(8 \mathrm{mV} /$ $\mu m$ ). (High sensitivity (2V/mil) proximity transducers are used in rolling element malfunction diagnostics; flaws on rolling elements or rings generate specific outer ring deflections observed by the transducer.) Proximity transducers provide vibration and static data. They cover the frequency range from zero to about $600 \mathrm{kcpm}$. The proximity probe requires an external power supply (usually -18 to $-24 \mathrm{Vdc}$ ) for operation. For signal accuracy, the shaft surface must be conditioned. The proximity probe is considered the best for measuring rotor lateral and axial vibrations and positions on rotating machinery. It is prized for easy calibration check, reliability, and robustness in the industrial environment. The proximity probes successfully replaced obsolete shaft riders.

2. Velocity transducer. The principle of operation of this transducer is based on inertial (seismic) property of a heavy mass suspended by springs on a vibrating body to remain still. In the electromagnetic transducer design, the mass carries a coil of wire and is elastically suspended by soft springs in a case containing in the middle a permanent magnet (Fig. A2). The case, attached to a vibrating structure (such as a bearing cap or machine casing), transmits its vibration. The relative motion between the coil and the magnet generates an output voltage proportional to the instantaneous velocity of vibration. The transducer is self-generating, and does not 


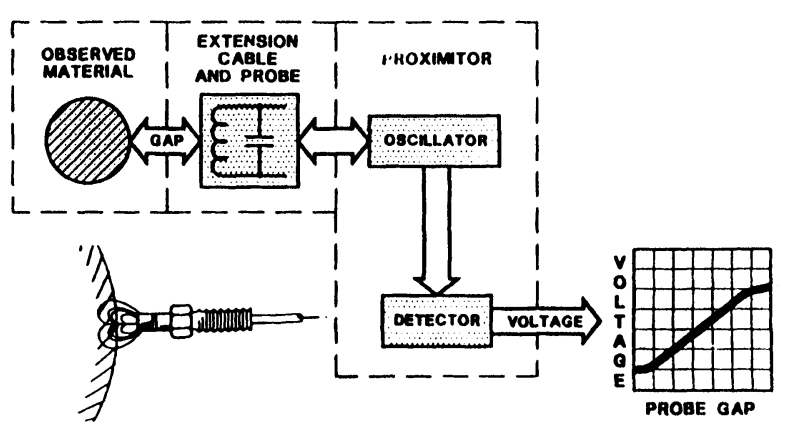

FIGURE A1 Eddy current proximity displacement transducer system: When a conductive material (shaft) approaches the probe, the voltage output becomes more positive.

require external power supplies. Velocity pickups have good sensitivity, typically 0.1 to $1 \mathrm{~V} / \mathrm{in} / \mathrm{sec}$ ( 4 to 40 $\mathrm{mV} / \mathrm{mm} / \mathrm{s}$ ). Their frequency range is from $500 \mathrm{cpm}$ to $1000 \mathrm{kcpm}$.

Velocity pickups are externally installed, and serve for overall vibration measurements of general purpose machinery. Disadvantages of velocity pickups comprise difficulties in calibration checks, sensitivity to magnetic interference, mounting orientation, and cross-axis vibration. The moving parts increase the risk of damage by a sudden shock or by fatigue process. More modern velocity pickups which do not contain moving parts are based on the same principle as accelerometers, and they include an electronic integration circuit.

3. Accelerometer. An accelerometer consists of an inertial mass mounted on a force-sensing element, such as a piezoelectric crystal (Fig. A3). The latter produces an output proportional to the force exerted on the inertial mass, which is, in turn, proportional to the acceleration of a machine component to which the transducer is attached. Typical sensitivity of an accelerometer is 0.1 $\mathrm{V} / \mathrm{g}$. Accelerometers are small, lightweight transducers that operate over a broad frequency range, as well as temperature range. They can withstand high vibration levels. Accelerometers do not require power supplies, and they are externally installed. They are, however, sensitive to a method of attachment and the surface condition. They are also sensitive to noise and spurious vibrations (some models contain an integrally mounted amplifier). Accelerometers serve the best for high frequency vibration measurements in the ranges from 1500 cpm to $1200 \mathrm{kcpm}$.

4. Dual transducer. Combination transducers are designed to measure the absolute motion of the rotor in space, as well as the motion relative to the machine housing. If the movement of the latter is larger than $30 \%$ of the rotor motion, the absolute vibration of the rotor

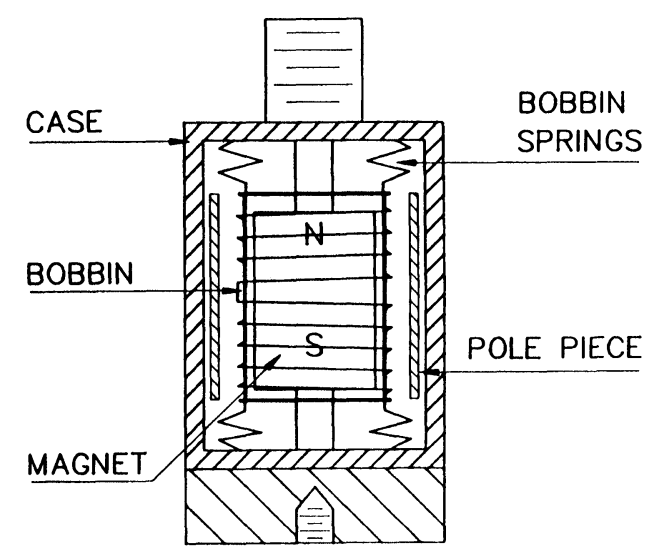

FIGURE A2 Electromagnetic velocity transducer.

should be known to adequately assess the machine health.

A dual transducer typically contains a proximity transducer and a velocity pickup (Fig. A4). The velocity signal represents the housing absolute motion. It is electronically integrated and summed with the signal from the proximity transducer to represent the rotor absolute displacement.

\section{Transducer Selection}

A selection of transducers for the machine monitoring system depends on the machine construction, estimated types of vibrational malfunctions and parameters which assess the malfunction, machine internal and external environment, rotative speed range, and the expected machine dynamic/vibrational behavior. The machine structure imposes limitations on the transducer installation. The environmental parameters, such as temperature, working fluid pressure, corrosiveness, and/or radiation indicate the transducer operational conditions. The expected machine dynamic behavior and its possible malfunction types answer the questions regarding what parameters to measure, what are vibration signal levels, signal-to-noise ratio, and frequency range.

It has to be well understood that the rotor of any rotating machine represents a source of vibration. By measuring the rotor vibrations, the direct information is obtained. When measuring casing vibrations using velocity pickups or accelerometers, the vibrational information is indirect, distorted by casing transmissibility. It is also incomplete, as rotor orbits cannot be obtained, and the signal resolution in the low frequency range is poor.

A selection of transducers and further data management systems can be made on a broad basis, generally, by dividing rotating machinery into categories, such as 


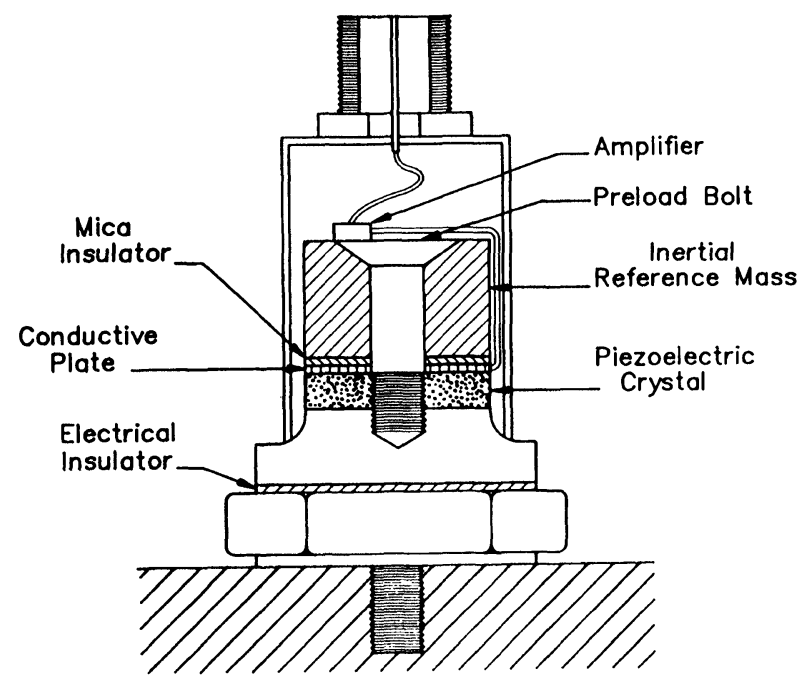

FIGURE A3 Piezoelectric accelerometer.

"critical," "essential," and "balance-of-plant" (general purpose machines). Large and expensive machines which cannot be spared, as well as those machines which would create a major hazard or production loss if they suddenly became inoperative, are classified as critical machines. The main factor is, therefore, a vulnerability of production to failure of a given machine. The critical machines have to be carefully instrumented with the best on-line systems. On the other hand, the easily replaceable general purpose machines may be periodically monitored, with acceptable results, by portable instruments.

\section{Machine Operating Modes for Data Acquisition}

The machine vibration and process data are collected during the following states:

(a) At rest: The data which is referred to as static data provides the rotor static position within the bearings, and may also reveal a presence of any external source of vibration. At machine rest, the structural resonances of various machine elements and adjoining constructions, such as pipelines, can be tested, using modal analysis methods.

(b) Slow roll, i.e., at low speed (typically less than $10 \%$ of the first balance resonance speed). In this condition, the rotor dynamic response is mainly due to rotor bow and/or electric and mechanical runout. The slow roll data serves for the rotor straightness check, and for the transducer/rotor surface conditioning check-up. The slow roll data are vital in the machine balancing process (Fig. A5) and in shaft crack diagnosis.

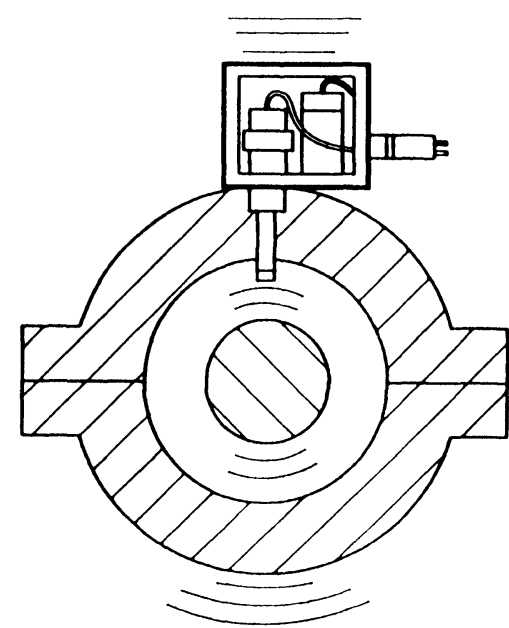

FIGURE A4 Dual transducer measuring casing absolute motion, and shaft relative and absolute motions.

(c) Start-up: Vibrational data captured during this transient state is extremely important. It helps to identify resonant speeds, vibration modes, slow-roll speed range, presence of self-excited vibrations, and provides information on modal effective damping and synchronous amplification factors. The best data is obtained if the start-up angular acceleration is small enough for good resolution of data versus rotative speed and low contamination by transients. The data display formats are polar and Bode plots of filtered components (Figs. 2, 9, 20, 23, and A5), shaft centerline position (Figs. 15 and A6), and spectrum cascade (Figs. 7, 8, 12, 13, 14, 16, 18, 19, 22, and A7). The plots may be accompanied by sequences of shaft orbits and timebase waveforms.

(d) Operating speed, i.e., at dynamic equilibrium of the machine: The vibration information referred to as steady-state data is most meaningful when processed using time-trend formats in order to assess any deterioration in the dynamic behavior. The data monitored at the operating speed can be displayed in the timebase waveform, orbit, spectrum (Figs. 4, 6, 7, 12, 15, 16, 20, A8), and in trend formats, such as amplitude and phase of filtered vibration components versus time (in "Bode" amplitude-phase-time (APHT) or polar formats (Fig. 21)). Overall vibration amplitude trend (Fig. A9), waterfall spectrum (Fig. A10), and shaft centerline position trend (Figs. 6, 17, and A11) provide also meaningful information. At operating speed, the nonsynchronous perturbation testing can be performed, in order to identify rotor Nonsynchronous Dynamic Stiffness, modal parameters, nonsynchronous amplification factors, and stability margins. This testing requires special additional devices. 


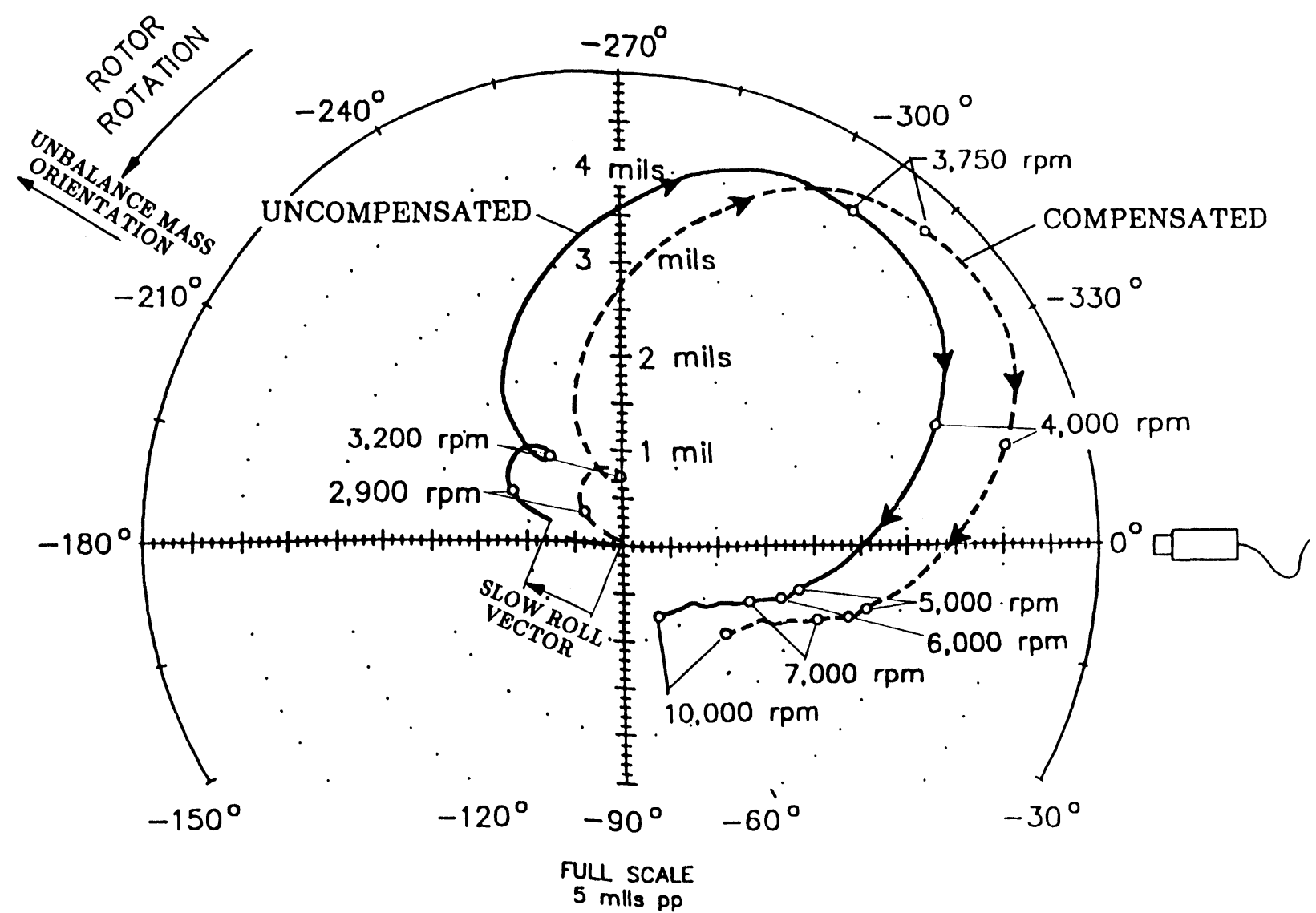

FIGURE A5 Polar plots of rotor uncompensated and compensated (slow roll vector subtracted) synchronous (1×) vibration data measured by a horizontally mounted displacement transducer.

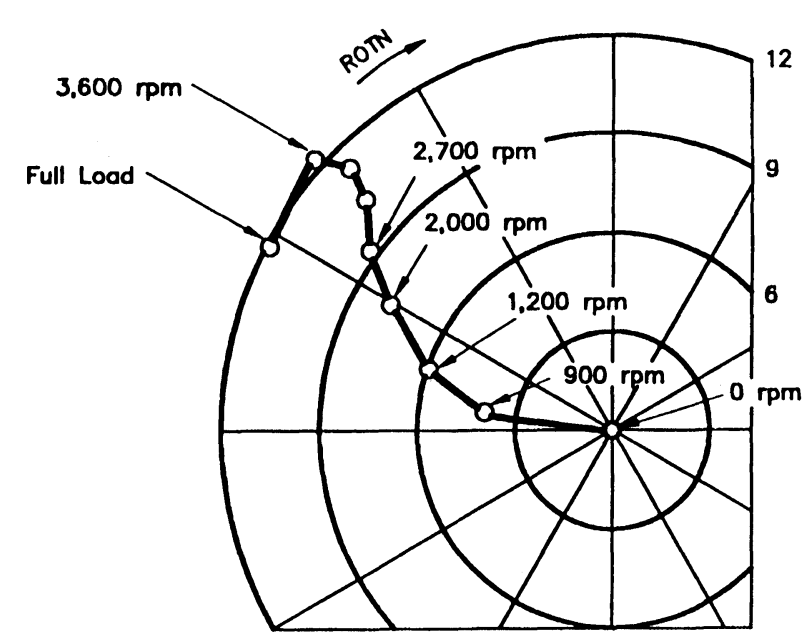

FIGURE A6 Shaft centerline position measured by XY probes and plotted versus rotative speed and load.

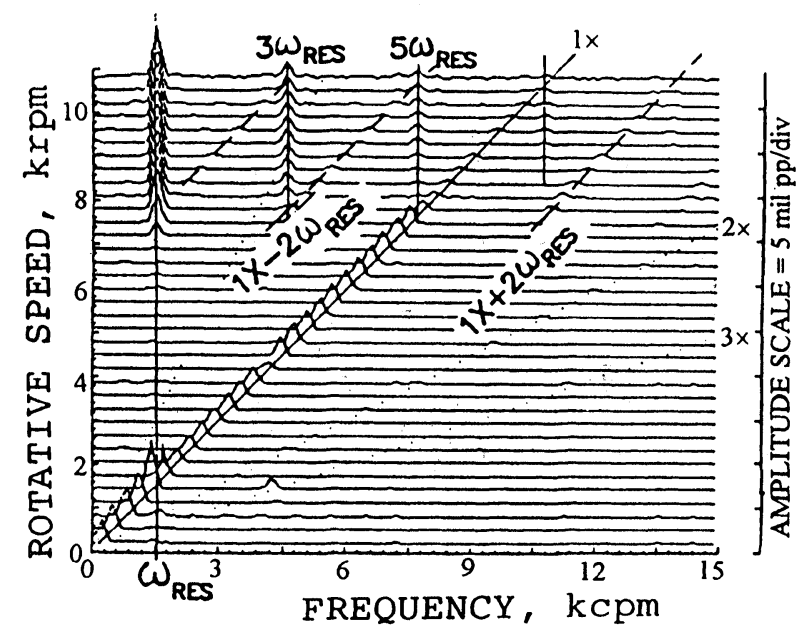

FIGURE A7 Spectrum cascade plot of rotor vibrations measured by a vertical displacement transducer. Presence of $1 \times$ and whip vibrations. Odd higher harmonics and sum/difference harmonics are also present. 
UNFILTERED

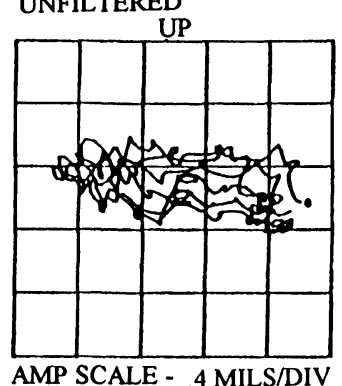

ROTATION: CW

$1 X$ FILTERED

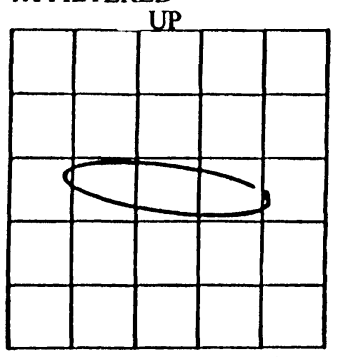

AMP SCALE - .4 MILS/DIV

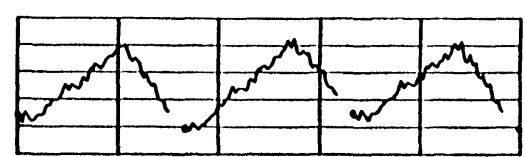

PLANT ID

TRAIN ID

MACHINE ID:

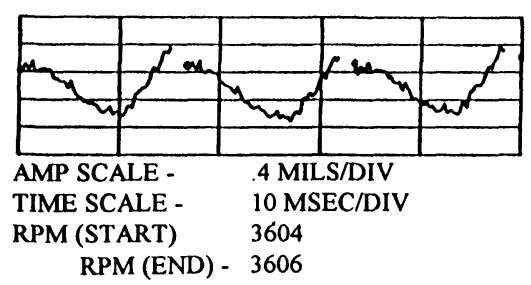

RUN:

DATE:

TIME:

PROBE \#1 ID:

ORIENTATION-

MAX AMP-

PROBE \#2 ID:

ORIENTATION-

MAX AMP-
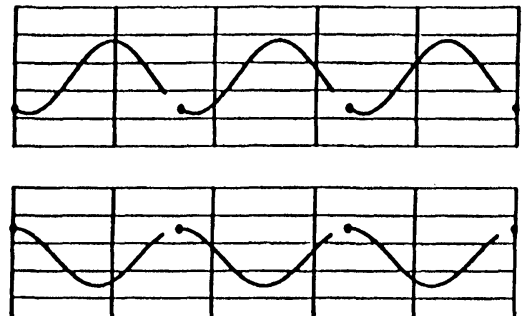

RUN:

DATE: $\quad 21$ MAY 81

TIME: 1208 Hour

PROBE \#1 ID: \#2 BRG VERTICAL

ORIENTATION- 135 DEG

IX VECTOR- $\quad 1.05$ MILS PK-PK

0 - 201

ORIENTATION- 45 DEG

IX VECTOR- $\quad .83$ MILS PK-PK

AMP SCALE - $\quad .4$ MILS/DIV

TIME SCALE - $\quad 10 \mathrm{MSEC} / \mathrm{DIV}$

RPM (START) - $3606 \quad$ RPM( END) - 3605
XYZ UTILITIES

TURBINE GENERATOR

1206 Hour

\#2 BRG VERTICAL

1.35 MILS PK-PK

BRG HORIZONTAL

45 DEG

1.07 MILS PK-PK

$0-356$

FIGURE A8 Unfiltered and $1 \times$ filtered timebase orbits of a rubbing turbine generator.

(e) Shutdown is, for some machines, the only practical mode for transient-state data acquisition. The shutdown data differs from the start-up data by the driving torque effects (absence/presence), and often by thermal and alignment conditions of the machine. The data can be displayed using the same formats as for the start-up data. During start-up and shutdown, the synchronous perturbation testing can be performed in order to identify the Synchronous Dynamic Stiffness and lowest mode modal parameters of the rotor.

\section{Keyphasor Functions, Phase Measurements, and Torsional Vibration Measurements}

The Keyphasor ${ }^{\circledR}$ is a radially mounted proximity transducer that observes a key, keyway, or other once-per-turn discontinuity on the rotor surface. During rotor rotation, the transducer generates a once-per-turn on/off-type signal (Fig. A12). This signal provides the rotative speed measurements and the reference for filtered vibration signal phase measurements. The absolute phase on a filtered signal is measured as a phase "lag" from the start of a blank Keyphasor dot to the first positive peak of the signal. The phases of filtered vibration components represent one of the most important diagnostic tools in rotating machinery. The Keyphasor signal serves, also, for the evaluation of vibration-to-rotation frequency ratio (Fig. A13).

Rotor torsional vibrations can be measured by a proximity transducer observing equally spaced, toothlike markers on the rotor (typically 36 markers). An instantaneous phase of the each tooth on/off signal is then subtracted from the "ideal" phase based on the constant rotative speed. An additional subtraction of signals from two such transducers mounted at different axial locations provides rotor torsional vibrations. In order to eliminate the effect of lateral vibrations on torsional responses, at each location, one more transducer is mounted at 180degree position. Data from two transducers are then added, so for the torsional vibration measurements, at least four transducers are required.

\section{Measurement and Documentation Conventions}

Conventions are designed to follow specific routines so that clarity and consistency of the data are maintained.

1. Cables should be properly identified so that transducer signals are not intermixed or inverted. 


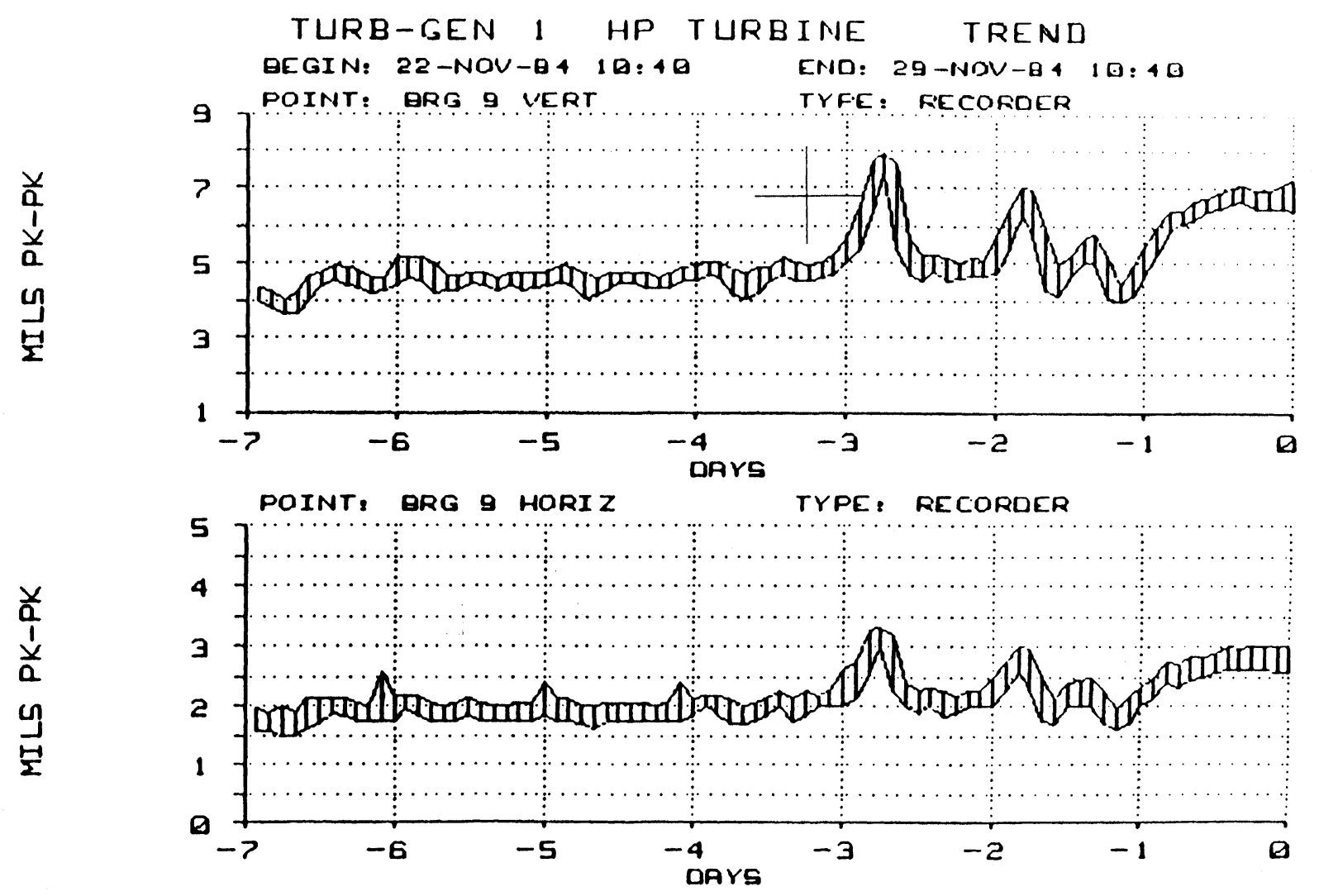

\begin{tabular}{|c|c|c|c|c|}
\hline IS FLFIIT & H HFRSELECT & D. CHE ORY & K3 FOLRR MEEKS & KA TWELUE WEEKE \\
\hline 15 ELEFEEIIT:IFL & & L.P LJET & KB PRIHIT & \\
\hline
\end{tabular}

FIGURE A9 Overall maximum and minimum amplitude trend.

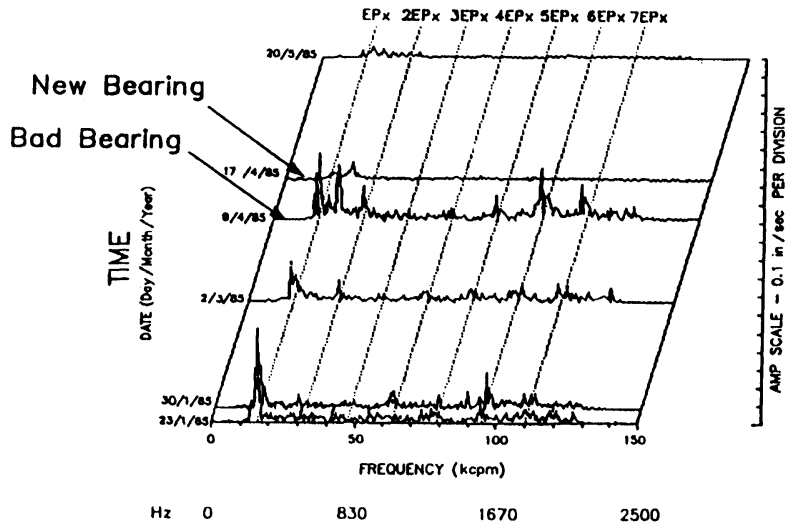

FIGURE A10 Waterfall spectrum of vibration response at operating speed of a rotor supported in rolling element bearings. $\left(E P_{x}\right.$ is element passage rate.)

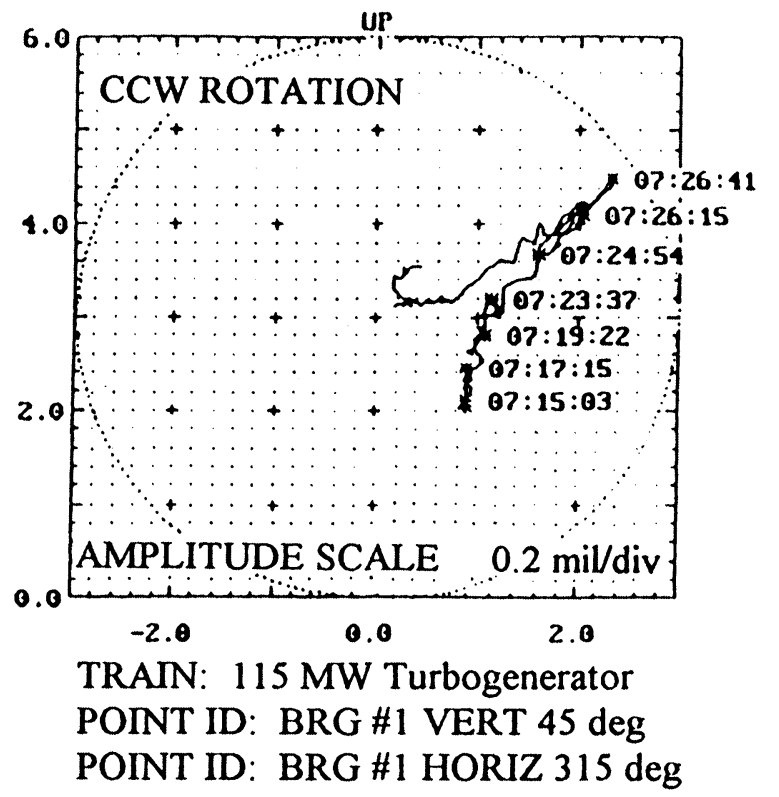

FIGURE A11 Shaft centerline position trend plot measured by XY displacement transducers. 


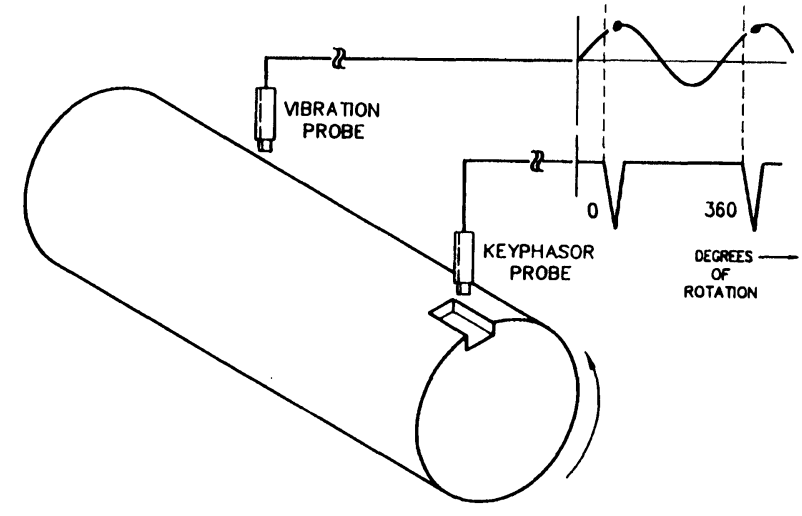

FIGURE A12 Keyphasor® once-per-turn reference.

2. Signal polarity: an important convention to ensure the correct signal polarity reads as follows: "Motion towards the transducer causes a positive voltage and/or current change" (Fig. A1).

3. Transducer orientation on the rotor:

(a) Radial proximity transducers: Two transducers should be mounted orthogonally to each other and orthogonally to the rotor axis (Fig. A14). Looking from the driving end, the "vertical" probe is designated as being 90 degrees in counterclockwise direction from the "horizontal" probe, independently from the direction of shaft rotation. The latter is determined when observed, also, from the driving end (Fig. A15).

(b) Axial proximity transducers: For thrust measure-

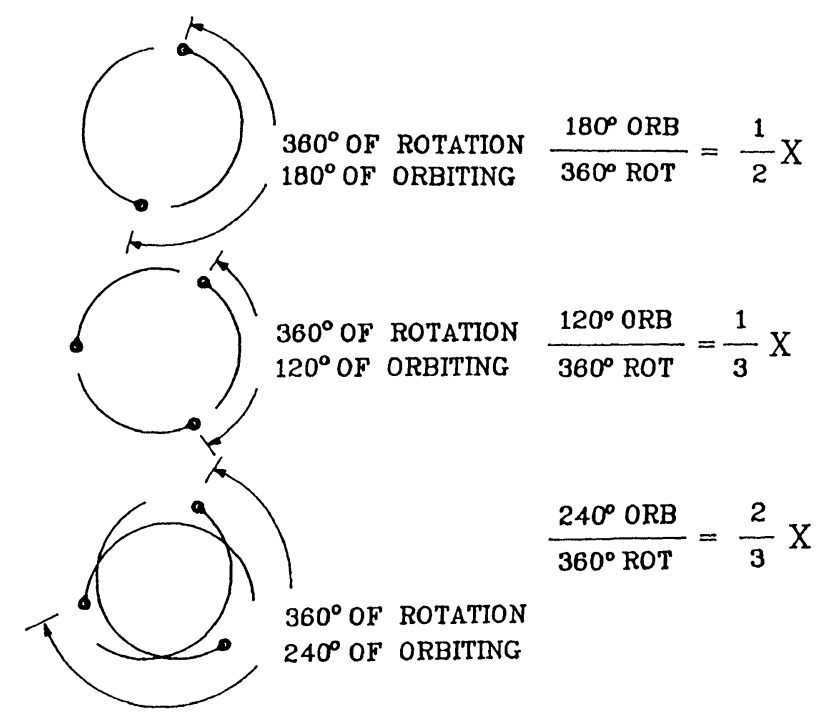

FIGURE A13 Vibration frequency estimation using Keyphasor marks on the orbits.

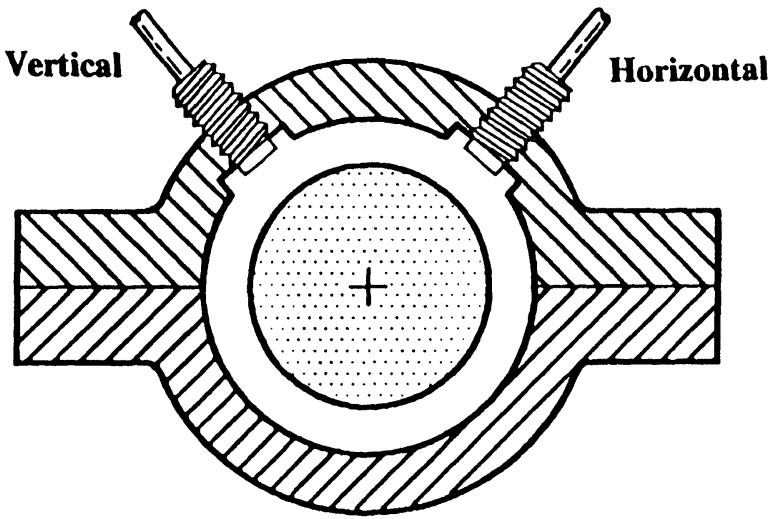

FIGURE A14 Displacement transducers mounted in XY orthogonal configuration.

ment, proximity transducers should be mounted within 12 inches $(30.5 \mathrm{~cm})$ of the thrust collar. One should observe the collar directly; if it is not an integral part of the shaft, the other observes the shaft (Fig. A16).

4. Oscilloscope convention (Fig. A17): A vertical probe signal corresponds to "Y" channel on the "up" side of the oscilloscope. A horizontal probe signal corresponds to channel " $\mathrm{X}$ " or the right side of the oscilloscope. Keyphasor probe signal connects to the "external input" (intensity axis " $Z$ ").

5. Plant conventions refer to specifics in documentations (Figs. A15 and A18). The documentation should include machine train diagram, machine dynamic specifications, machine/component construction data, instrumentation description, conventions, and dynamic (baseline/historical) reference data.

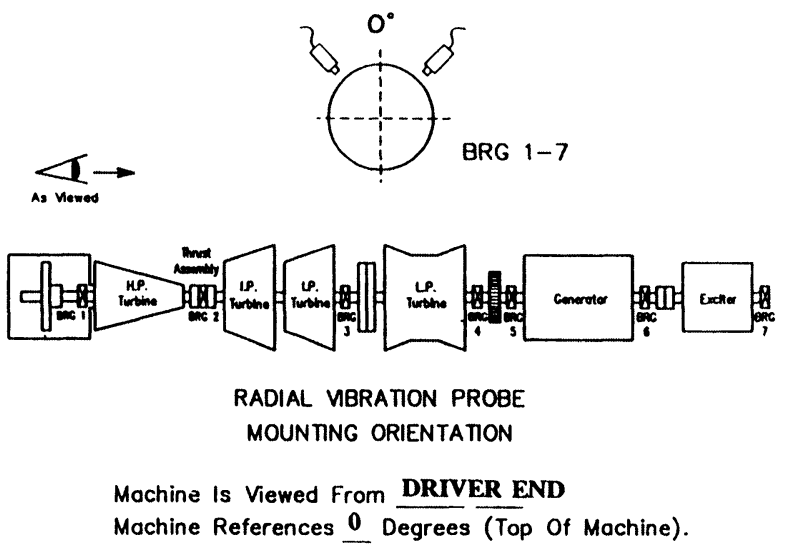

FIGURE A15 Turbogenerator diagram with lateral vibration displacement transducer orientation. 


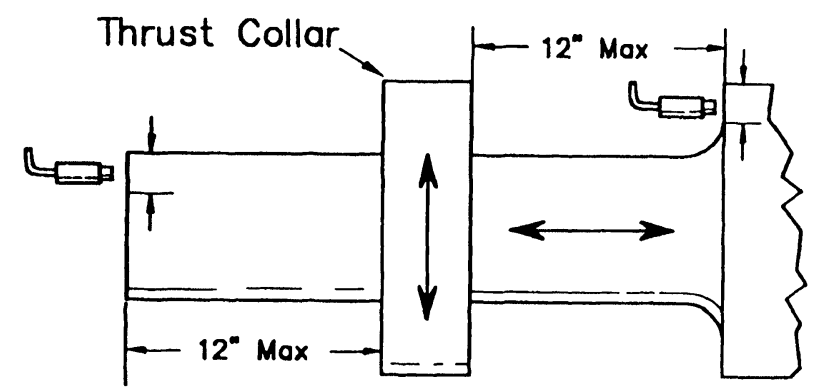

FIGURE A16 Axial (thrust) position/vibration measurements.

\section{Recommendations for Monitoring}

Monitoring of rotating machines includes a broad range of protection and information systems. On general purpose machines, vibration probes and temperature sensors are installed primarily to protect the machine from catastrophic failure. The transducers can provide, however, much more useful information which can be used in early diagnosis of an impending malfunction and in preventing the failure. The recommendation for vibration and temperature monitoring is as follows:

- Two shaft observing proximity probes mounted in XY orientation at or in proximity of each bearing.

- Two proximity probes for monitoring shaft axial position/vibration. Axial position is an extremely important measurement, because it indicates changes in machine internal clearances or thrust reversals which may lead to catastrophic consequences on the machine. As a result, many users tie axial position to a main trip function.

- A Keyphasor probe.

- Redundant probes for any of the above which are inaccessible from the machine exterior.

- Two additional XY proximity probes at the opposite sides of each bearing for mode identification, and in

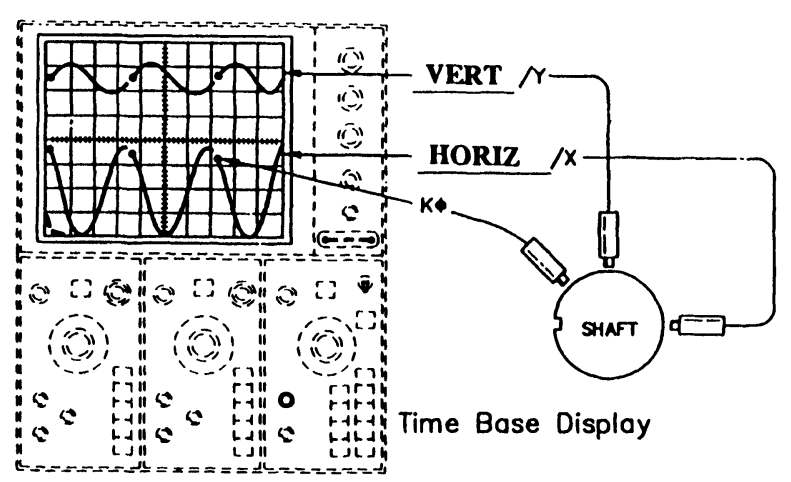

FIGURE A17 Oscilloscope convention.

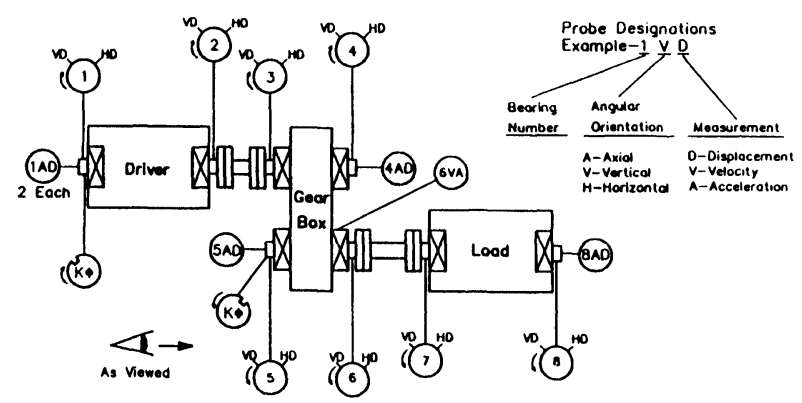

FIGURE A18 Transducer orientation on a turbine-driven compressor.

order to avoid a lack of signal from a probe located in a nodal point for some modes. (These "modal" probes can also be mounted on the same sides of the bearings, but at least one shaft diameter distance from the other probes.)

- Two temperature sensors located in each radial bearing for short bearings (with length-to-diameter ratio less than $1 / 2$ ) one sensor will be sufficient.

- Two temperature sensors mounted in active and inactive faces of the thrust bearing.

\section{Instruments for Data Processing and Displaying in Real Time}

1. Oscilloscope. The oscilloscope is one of the most important instruments for visual observation of the vibration signals in real time. It displays the amplitude, frequency, phase, and, if available, position data simultaneously. The shape of the waveform or orbit can highlight rotor response significant changes that can goundetected when these characteristics are viewed separately. The orbit is especially meaningful for the data provided by the shaft observing XY proximity transducers, and the Keyphasor transducer. The orbits display actual magnified paths of the rotor centerline. The Keyphasor dots correlate rotative speed with the shaft vibrational motion, and give an idea about the shaft mode of vibration, when orbits with Keyphasor dots from two ends of the shaft are compared (Fig. A19).

2. Monitors. Monitors are designed to continuously monitor and display a wide variety of supervisory parameters measured by transducers. They aid operation personnel in recognizing machinery problems, and automatically shut down machines before costly damage due to malfunction occurs (Fig. A20). The monitors are equipped with "OK", "Alert", "Danger" indicators and "Alarm" relays. The monitors are usually built in modular versions to choose elements for specific machine needs. Many of them are equipped with possible computer interfacing. 


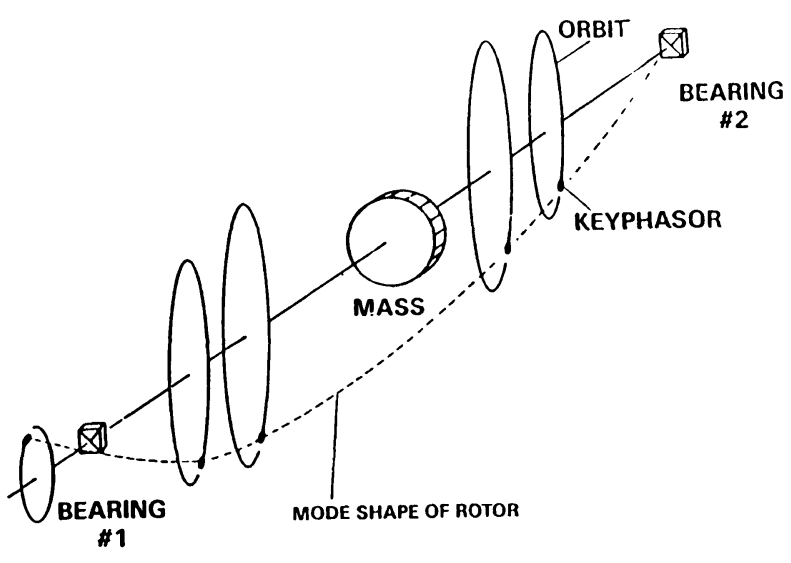

FIGURE A19 Shaft mode shape determined from orbit and Keyphasor mark information.

3. Filters. In the frequency domain, filters serve to focus on specific vibration components or eliminate unwanted components (such as noise) of the transducer signal (Fig. A21). A high or low pass filter eliminates, respectively, the low or high frequency components of the signal. In the rotating machinery applications, the most useful is the tracking filter, such as a Digital Vector Filter. This instrument automatically adjusts a narrow bandpass filter center frequency to the frequency of a reference signal, usually that of the Keyphasor signal.
The tracking filter can thus provide the filtered synchronous vibration $(1 \times)$, as well as vibration signal components being fractions or multiples of the rotative speed $(1 / 3 \times, 1 / 2 \times, 2 \times, 3 \times$, etc. $)$.

If the bandpass filter is swept across the frequency range (sweep filter), a spectrum will be generated. It serves for identification of significant frequency components of a vibration signal.

4. FFT Spectrum Analyzer. This instrument is widely used (and often misused) in vibration analysis. It provides the Fourier spectrum of the vibration signal. In rotating machine diagnostics the most often used are steady-state spectra, spectrum waterfalls versus time, or versus some other monitored parameter, and spectrum cascade plots versus rotative speed from the machine start-up or shutdown. For rolling element bearing and gear train diagnostics, however, the spectrum analyzer is often the primary measuring instrument. The spectrum analyzer is also used for identification of the instability source location along the rotor of a machine train (phase measurement of cross-correlated signals, such as fluid whip, from two ends of the rotor).

\section{Computerized Data Acquisition and Processing Systems}

Vibration and process data from machines can be visually monitored, and/or manually acquired and reduced

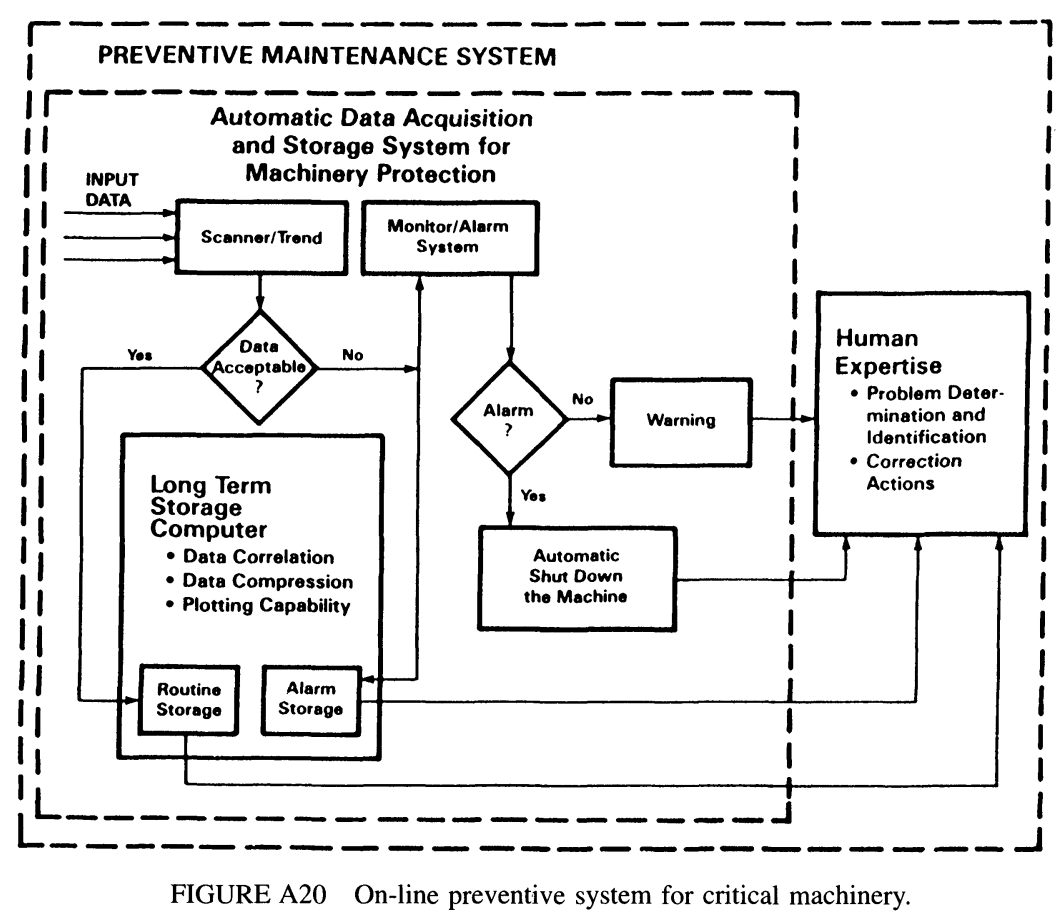




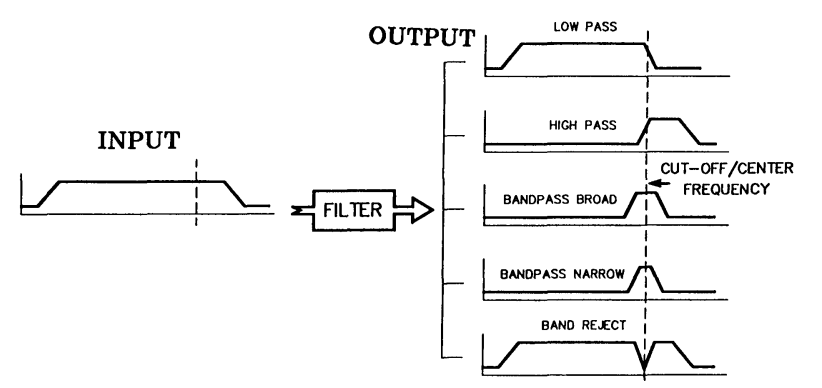

FIGURE A21 Filter types.

ware provides data processing, displaying and archiving routines (Fig. A22).

The computer can perform many of the data processing functions previously described, thus replacing a portion of hardware. Computers can provide the processed data in almost real time, but their most important advantage may lie in their ability to store data for further analysis, especially in correlation with other operating parameters, and in comparison with previous, "histori-

into meaningful for mats. The advances in computer technology have brought tremendous improvements in increasing accuracy, as well as in reducing time and effort consumed by data acquisition and processing. The improvements are also in the volume and speed of performed operations, from the beginning of data collection, to the final display of the data now available in cross-correlated formats. Decreasing costs of computerized data acquisition/processing systems, and their efficiency, result in increasing cost-effectiveness of their application in industry maintenance problems.

A typical system consists of a computer and a data acquisition instrument, which digitizes the transducerprovided electronic signals. A dedicated computer softcal" data.

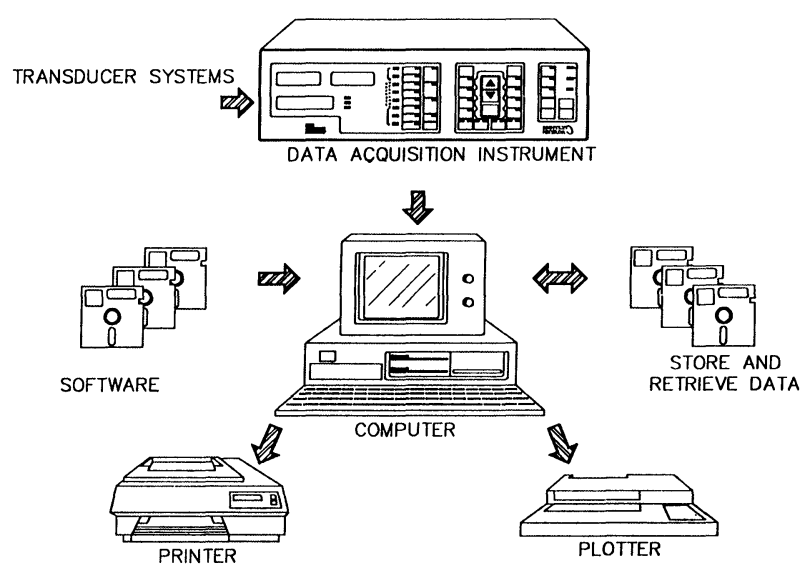

FIGURE A22 Computerized data acquisition and processing.

FIGURE A22 Computerized data acquisition and processing. 

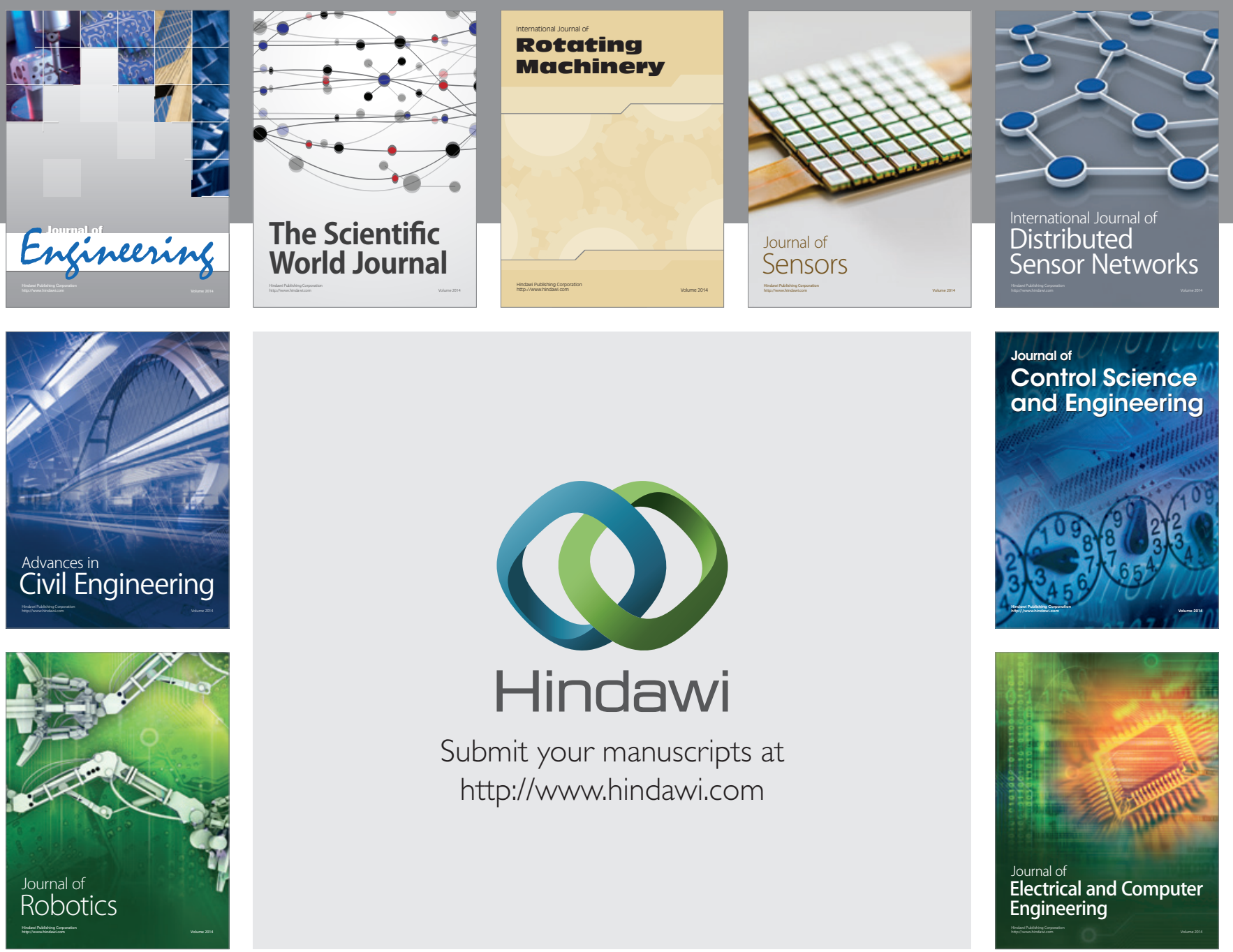

Submit your manuscripts at

http://www.hindawi.com
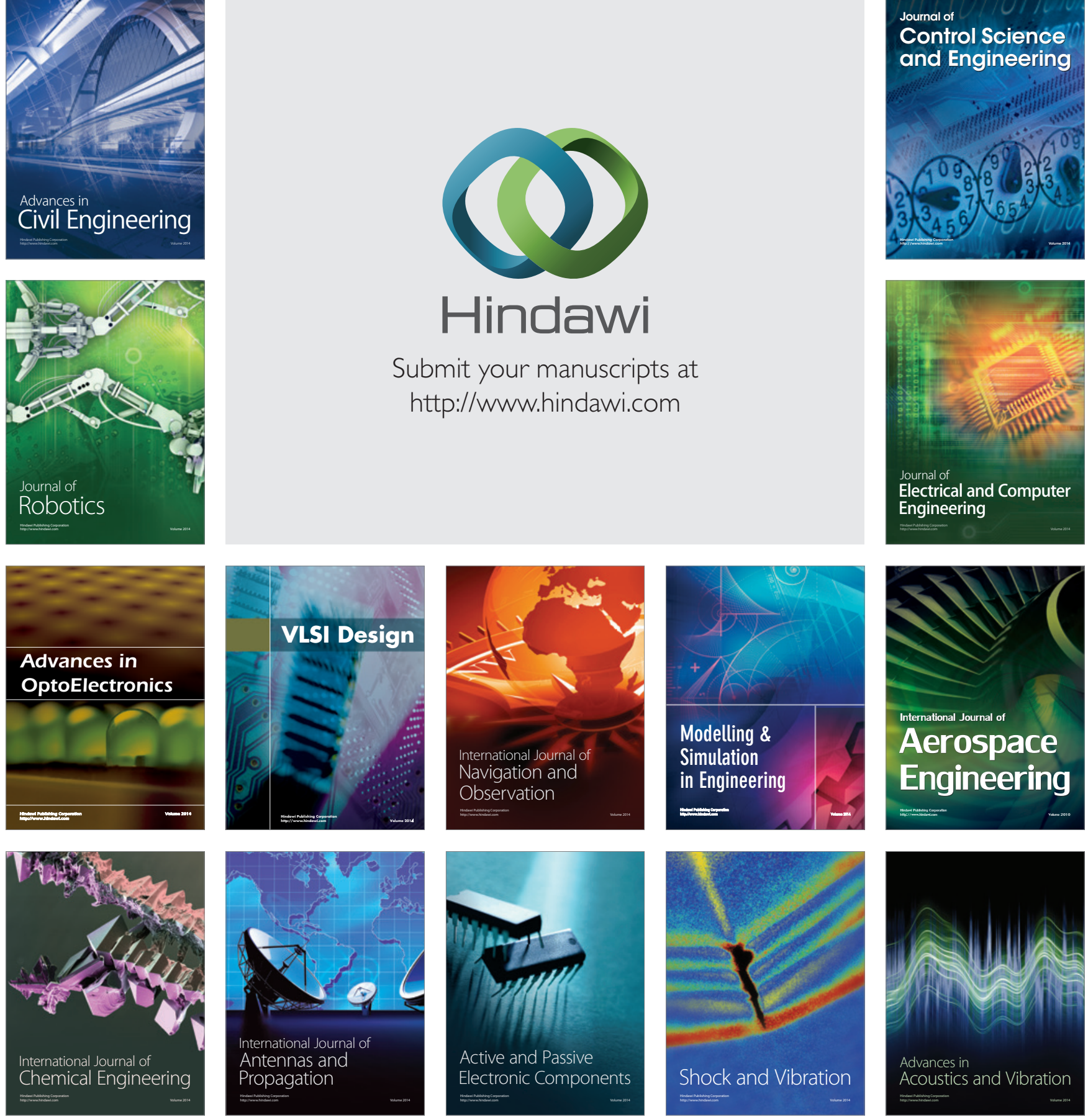